\title{
Applying Many-Facet Rasch Modeling in the Assessment of Creativity
}

\author{
Ricardo Primi ${ }^{12}$ \\ Paul J. Silvia ${ }^{3}$ \\ Mathias Benedek ${ }^{4}$ \\ Emanuel Jauk ${ }^{4}$
}

${ }^{1}$ Post Graduate Program in Psychology, Universidade São Francisco, Campinas, Brazil, ${ }^{2}$

EduLab21, Ayrton Senna Institute, São Paulo, Brasil, ${ }^{3}$ Department of Psychology,

University of North Carolina at Greensboro, United States , ${ }^{4}$ Department of Psychology, University of Graz, Austria,

Draft version 1.0 of 5/22/2018. This paper has not been peer reviewed. Please do not copy or cite without author's permission.

The first author also received a scholarship from the National Council on Scientific and Technological Development (CNPq). Correspondence should be addressed to: Ricardo Primi, Universidade São Francisco, Rua Waldemar César da Silveira, 105, Campinas, São Paulo, CEP 13045-510; e-mail: rprimi@mac.com 


\begin{abstract}
Creativity assessment with open-ended production tasks relies heavily on scoring the quality of a subject's ideas. This creates a faceted measurement structure involving persons, tasks (and ideas within tasks), and raters. Most studies, however, don't model possible systematic differences among raters. The present study examines the impact of individual rater differences in the reliability and validity of creativity assessments. It applies the Many-Facet-Rasch-Model (MFRM) to model and correct for these differences. We reanalyzed data from two studies (Ns=132 and 298) where subjects produced metaphors, alternate uses for common objects, and creative instances. Each idea was scored by two to four raters. We simulated several conditions of reduced load on raters where they scored subsets of responses. We then compared the reliability and validity of IRT estimated scores (original versus IRT adjusted) on various conditions of missing data. Results show that (a) raters vary substantially on the lenient-severity dimension, so rater differences should be modeled; (b) when different combinations of raters assess different subsets of ideas, systematic rater differences confound subjects' scores, increasing measurement error and lowering validity; and (c) MFRM adjustments effectively correct for rater effects, thus increasing validity. We conclude that MFRM is a powerful means to model rater differences and reduce rater load in creativity research.
\end{abstract}

Keywords: creativity, assessment, many facet Rasch model, raters, planned missing data, validity, reliability 


\section{Applying Many-Facet Rasch Modeling in the Assessment of Creativity}

The creativity of an idea or product is necessarily subjective (Sawyer, 2006), so researchers commonly assess creativity by asking a group of judges to assign creativity scores (Kaufman, Plucker, \& Baer, 2008). This seemingly simple approach, however, raises some complex methodological issues. How should reliability be quantified for complex, faceted designs, such as several judges rating several items for several creativity tasks per person? How can differences in leniency or severity between the judges be measured and corrected for? Must every judge score every task for every participant, or can efficient designs be employed?

To date, the methods commonly applied to subjective ratings in faceted designs solve some but not all of these problems. In this article, we describe many-facet Rasch models (MFRM; Eckes, 2011; Linacre, 1994), an extension of Rasch modeling for faceted data. We illustrate how these models can handle three major needs for subjective scoring: they yield a holistic measure of reliability; they quantify and adjust for each rater's severity; and they afford efficient planned missing designs that greatly reduce rater burden. Using two illustrative datasets, we describe how to conduct and interpret many-facet Rasch models in R (R Core Team, 2016) and examine the influence of missing ratings on reliability.

\section{Subjective Ratings in Creativity Research}

Subjective ratings are widespread in creativity research. The basic approach involves having raters assign quantitative scores that reflect the creative qualities of ideas and products. In lab research, many tasks designed to measure creative cognitive processes rely on subjective scoring. In their early work, Guilford and his group had raters score ideas for dimensions like cleverness and remoteness (e.g., Christensen, Guilford, \& Wilson, 1957). Divergent thinking tasks - perhaps the most popular lab tasks in creativity research — are often scored for overall creativity (Silvia et al., 2008) or for specific dimensions, such as 
novelty, appropriateness, or realism (Cropley \& Kaufman, 2012; Diedrich, Benedek, Jauk, \& Neubauer, 2015). Humor production tasks ask participants to come up with funny ideas on the spot—-such as writing funny captions for single-panel cartoons — and then have raters judge the funniness of the captions (Nusbaum, Beaty, \& Silvia, 2017). The Consensual Assessment Technique (CAT; Amabile, 1982) is probably the best-known approach to subjective scoring. The CAT asks experts in a domain to rate the creative quality of products according to their personal definitions of creativity. Subjective ratings of creativity are prominent outside of lab research as well. For example, the creative and expressive aspects of musical performance (Ruthsatz, Detterman, Griscom, \& Cirullo, 2008), the creative quality of paintings (Hekkert \& van Wieringen, 1996) and moving image art (Benedek et al., in 2017), the creativity of architects' bodies of work (MacKinnon, 1962), and the artistic importance of works in the classical music canon (Kozbelt, 2005) have all been assessed by asking judges (typically experts) to assign quantitative ratings.

\section{Challenges in Subjective Ratings}

Although popular, subjective ratings bring some underappreciated statistical and methodological problems. First, studies using ratings typically have a faceted data structure: for example, a sample of 150 participants will complete 3 divergent thinking tasks, and 4 raters will score every participant's response to every task. As a result, evaluating the reliability of the assessment design is not straightforward. Researchers typically ignore a facet. For example, they will report the internal consistency of the three tasks, omitting variation due to raters, or report the agreement of the raters, omitting variation due to tasks.

Methods anchored in generalizability theory (Cronbach, Gleser, Nanda, \& Rajaratnam, 1972) can provide a holistic estimate of reliability for faceted designs, and they have been useful in creativity research (e.g., Kaufman, Lee, Baer, \& Lee, 2007; Long \& Pang, 2015; Silvia et al., 2008, Study 1). But they don't solve the second challenge: 
estimating and correcting for rater differences. Not surprisingly, raters can vary substantially when asked to judge how creative, funny, clever, or interesting an idea is. No matter how extensively they are trained or how detailed the scoring rubric is, some raters will always be more lenient and other will be more severe (Eckes, 2011). Generalizability theory can estimate the amount of variance attributable to a rater facet, but it won't scale each rater on an underlying severity dimension or correct a participant's estimated trait score in light of the raters' severity.

And third, subjective scoring methods impose a heavy burden as sample sizes increase (Forthmann et al., 2017). Virtually all studies use complete-data rating designs: all raters rate all responses for all participants. The practical issues of rating thus constrain the sample sizes researchers can collect. If participants complete 4 tasks where they generate on average 5 responses or products that 3 raters score, for example, then adding 100 participants creates 6000 more judgments to be made and recorded. Recruiting large samples becomes impractical for such rating systems. If researchers decide to reduce this burden by having different raters score different subsets of responses, rater differences in leniency-severity becomes a critical issue because raw responses are not comparable when raters vary in severity. The model presented here is a way to equate judge scorings into a common scale, thus overcoming this problem.

\section{Many-Facet Rasch Models (MFRM)}

Many-facet Rasch models (Eckes, 2009, 2011; Linacre, 1994) are a potential solution. Many-facet Rasch models for polytomous scorings can be written, following Eckes (2009), as:।

$$
\log \left(P_{n i j k} / P_{n i j(k-1)}\right)=\theta_{n}-\beta_{i}-\alpha_{j}-\tau_{k}
$$

This equation expresses the $\log$ of the odds of person $n$ receiving a rating $k$ rather than $k$ - 1 on item $i$ by rater $j$. If a 4 -point rating scale is used ( 0 to 3$)$, this equation compares the 
odds of receiving 3 vs 2,2 vs 1 and 1 vs 0 . The interesting feature of this formulation is that the general odds of receiving any possible high rating — as compared to an immediate lower one-is a linear comparison of the subject $n$ latent ability theta $\theta_{n}$ versus the "difficulty" of the task, represented by a joint combination of the task difficulty $\beta$, rater $j$ severity $\alpha_{j}$, and the difficulty of receiving $k$ rating as compared to $k-1$. With four possible ratings ( 0 to 3 ) there are three thresholds $\tau_{\mathrm{k}}$ representing the difficulty of ratings 1 vs $0\left(\tau_{1}\right), 2$ vs $1\left(\tau_{2}\right)$, and 3 vs $2\left(\tau_{3}\right)$. Note that all these three parameters related to the task have a negative sign compared to theta, which has a positive sign. This means that the likelihood of having an idea that will be scored 3, for instance, is a fundamental comparison of subjects' creative ability versus the general difficulty of the task, the severity of the particular rater, and the global difficulty of score 3 . In this formulation the thresholds $\tau_{\mathrm{k}}$ are assumed to be equal for all tasks and also all raters, but this could be relaxed in more complex versions of MFRM models.

This MFRM formulation is an extension of the Rasch-Andrich Rating Scale Model called the three-facet rating scale model (Eckes, 2009; Wright \& Masters, 1982). Rating Scale Model includes parameters for subject, item, and ratings but not raters. MFRM extends this model by including the effect of differences in rater's severity. A very important consequence is that the latent trait parameter $\theta_{n}$ is not biased by rater's differences. For instance, if we use the rating scale model and we had different raters $A$ and $B$ scoring two disjoint subsets of responses from persons $a$ and $b$, and they differ in the severity dimension (A more severe than B), we would have only one parameter modeling the difficulty of the task in $a$ 's and $b$ 's subset of responses equations. Then the systematic rater differences will be reflected on $\theta_{n}$, that is, $a$-subjects will have latent $\theta$ scores systematically lower than $b$ subjects because of unmodeled rater differences. If we employ MFRM, however, this confounding will not happen because latent theta scores will be corrected for rater differences. 


\section{Correcting for Rater Severity}

In many-facet Rasch models, one can compute "fair average scores" that are a transformation of a subject's $\theta$ (and a rater's $\alpha$ ) back to the original metric (in this case, a 0 to 3 scale). The fair average is an adjusted expected score of a particular subject as if he or she had answered an average task that was scored by an average rater. When performing this adjustment, formula 1 is written as:

$$
\log \left(P_{n i j k} / P_{n i j(k-1)}\right)=\theta_{n}-\beta_{0}-\alpha_{0}-\tau_{k}(2)
$$

where $\beta_{0}$ is the mean difficulty for items and $\alpha_{0}$ is the mean of the raters' severity. A transformation of Equation 2 can produce expected category response probabilities for subject $n$ on each rating $r$ up to a maximum rating of $s=3$ (0 to 3$)$ denoted as $P_{n s}$. Then the fair average score is calculated as:

$$
M_{\text {fair } n}=\sum_{r=0}^{s} r P_{n s}
$$

So each score $r$ is weighted by the expected probability given subject's $n$ ability (and the difficulty of receiving a particular score), adjusting the other facets to an average rater and item, and then summed to result an average fair score for each subject.

\section{Accommodating Missing Data}

When using incomplete data, subjects will have their responses scored by different combinations of raters. Therefore, the unadjusted average scores of a subgroup of subjects will be affected by characteristics of a particular rater who happened to be assigned to score those subjects' responses. When two lenient raters are combined, for example, subjects' unadjusted score averages will tend to be higher as compared to the whole group. And more importantly this difference in rater behavior will be confounded with the subjects' ability. In an unlikely scenario of high absolute rater agreement, this problem is minimized since exact agreements are found across raters for most of the ratings (Stemer, 2004). But in a more typical scenario of moderate absolute agreement between raters, which can still go along with 
high relative agreement in terms of high internal consistency, rater differences will potentially affect unadjusted average scores when using incomplete data.

The most interesting feature of the adjusted scores from MFRM is that rater severity is explicitly modeled and therefore is no longer confounded with the examinees' ability. Therefore, it solves the problem of the comparability of subjects' scores when analyzing incomplete data. Subjects' theta scores are thus comparable across booklets that represent different combinations of raters for specific subsets of responses. This opens the possibility of reducing rater workload because not all raters need to rate all ideas - and not all tasks need to be answered by all subjects.

\section{The Present Research}

MFRM have a long history of successful application in various research areas (see http://www.winsteps.com/facetman/references.htm) but is relatively unknown in creativity research. We believe that the use of MFRM could improve current methods of assessing creativity. We thus reanalyzed data from two studies-Silvia and Beaty (2012) and Jauk, Benedek and Neubauer (2014) — in which raters give quality ratings for ideas produced in divergent thinking tasks (metaphor production, alternative uses, and instances of a concept/idea) using MFRM. Our main questions were (a) is there a noticeable difference in terms of leniency versus severity between raters? If it exists, does it have an impact on the quality of measurement? (b) Is there a practical advantage of using MFRM to model differences between raters? Is the use of MFRM justified in terms of improved reliability and validity? (c) Can we reduce the load on raters by doing an optimized missing data plan and then using MFRM to achieve a common metric between raters? In this case, how much can rater burden be reduced without reducing validity and reliability? Our final aim is to provide a practical tutorial in how to run MFRM using FACETS software and R.

\section{Study 1: Metaphor Ratings}


This study is a reanalysis of data from a study of creative metaphor production (Silvia \& Beaty, 2012). Participants generated two metaphors that were rated by three raters in a scale of 1 to 5 in the level of creativity. The primary goal in this reanalysis is to fit a MFRM model and explore the model parameters, especially raters' parameters. We also compared original scores with latent scores (i.e., the equated "fair average" scores) corrected for raters' differences. Finally, we introduced missing data by simulating a situation where not all raters rate all responses. We then explored the correlations of equated $v s$ original scores with the scores computed with full data set, as well as effects of missing data on validity coefficients.

\section{Method}

\section{Participants}

A total of 132 young adults (41 men, 91 women) enrolled at the University of North Carolina at Greensboro took part in the research and received credit toward a voluntary research option in a psychology course.

\section{Measures}

Metaphor production. The participants completed two metaphor tasks. After learning about definitions of metaphors and reading some examples, the participants were given two prompts and asked to complete them by creating a metaphor. The researcher emphasized that the study was about creative metaphors, so the participants should aim to come up with a metaphor that was creative, clever, and interesting.

The first prompt was "Think of the most boring high-school or college class that you've ever had. What was it like to sit through?"; the second prompt was "Think about the most disgusting thing you ever ate or drank. What was it like to eat or drink it?”. The participants were asked to come up with a single metaphor for each prompt. They could spend as much time as they wished on each one. To help spur responses, we provided some sample stems (e.g., "Being in that class was like...," "That class was..."). 
Each participant thus generated two responses. All responses were then judged by a group of 3 raters. The raters scored each response independently of each other. The responses were stripped of all identifying information, so the raters were unaware of any of the participant's other data or which two responses belonged to any particular participant. The raters gave a holistic creativity judgment for each metaphor based on a 5-point scale $(1=$ not at all creative, $5=$ very creative .

Intelligence and personality. To examine predictors of metaphor quality, we collected measures of fluid intelligence $(G f)$ and personality. $G f$ was measured using a group of 6 inductive reasoning tasks (see Silvia \& Beaty, 2012, for details). Personality was measured with the 60-item NEO FFI (Costa \& McCrae, 1992), which measures the five broad factors of personality. People completed the NEO FFI using a 5-point response scale $(1=$ strongly disagree, 5 = strongly agree) .

\section{Design and Statistical Modeling}

Each participant gave two responses that were rated by three raters. Therefore, we have six scored responses per participant, resulting in 786 data points (131 subjects X 6 responses). We used FACETS (Linacre, 2013), which implements several MFRM models using joint maximum-likelihood estimation method (JMLE) to compute the model parameters of Formula 1 and scores of average creativity using Formula 3. Reader will find a tutorial on how to run MFRM with the data of this study in FACETS and in R as a supplemental online material.

We ran models three times: (1) the benchmark with complete data, (2) then introducing $33 \%$ of missing data, keeping $67 \%$ of the total available data points (g67), and (3) then introducing $57 \%$ of missing data, keeping $43 \%$ of the total available data points (g43). For the g67 we ordered subjects randomly and then, for each subject, we eliminated data from one of the raters: for example, subject 1 had rater 1 data deleted, subject 2 rater 2 
data deleted, subject 3 rater 3 data deleted, subject 4 again rater 1 deleted, and so on. This group had a total of 524 data points. For the g43 we reproduced a common situation in research where we have a set of common responses scored by all raters and then for the remaining dataset each subject is scored by only one rater. We randomly selected 20 subjects to be the common responses for which we preserved the complete data. For the remaining 111 subjects we selected scored responses of only one rater, rotating raters so as to have equal number of subjects scored by each rater. This group had a total of 342 data points. We compared reliability and validity indices across these two levels of incompleteness.

\section{Results}

We first explore the model parameters of tasks and raters using the full dataset. The upper part of Table 1, in the first wo lines, shows average scores on each task, the fair average - the observed score corrected from other elements on the facets - beta parameters of task difficulty (and their standard errors), fit statistics (infit and oufit), and item-theta (latent score) correlations. The next three lines show the same information for raters, now called alpha parameters of rater's difficulty. Fit indexes are directly proportional to residuals, that is, the difference between observed versus expected scores. Values below 1.4 usually indicate acceptable fit (Linacre, 2013). The lower part of Table 1 shows classical inter-rater reliability measures based on absolute agreement (consensus measures): the percent agreement and the weighted Kappa index.

We observe that task difficulty is not so dispersed ( $\beta$ 's from -.20 to .20$)$ in comparison to raters, who had a wider range of severity ( $\alpha$ 's from -1.18 to .64). Fit statistics suggest a good fit, that is, no marked deviations from the expected model responses when we zoom in and analyze each particular rater's or task's residual separately. The rater-total (measure) and task-total (measure) correlation is high, above .50. The Rasch average reliability, which is a measure of internal consistency based on estimated parameters of 
Rasch model, was $r_{t t}=.46$. This is rather low when we compare with expected benchmarks of .70 as acceptable level of reliability. But this should be interpreted relative to the number of data points per subject (only six) and also considering that Rasch reliability is an estimate of the lower-bound of the true reliability value (Linacre, 2004). Raters' classical reliability indexes — agreement and weighted kappa — are presented in the lower part of Table 1 . These indexes show that in general raters have fair agreement (.25 to .39). Only J2 vs J3 had a lower agreement when considering exact scores. In general, we observed that raters' scoring behavior showed an acceptable level of agreement.

Figure 1 presents the distributions of all model parameters (subjects' ability, items difficulties, raters' parameters, and scale thresholds for score points). Since all parameters are on a common scale we can compare parameters with each other. A first noticeable result is that the distribution of creativity is positively skewed, that is, it is denser at the lower end (-1 to -4.5). At this level the most likely score, given by the right most figure, is 1 "not at all creative”. We noted also that the two items were equivalent in terms of difficulty. One of the raters was substantially more lenient than the other two. Although the rating scale spans from 1 to 5, no one received a score of 5. That's why in Figure 1 we see only scores of 1 to 4 .

\section{Insert Figure 1 here}

$$
\text { Insert Table } 2 \text { here }
$$

Table 2 shows the correlations under the three conditions of incomplete data (full, g67, and g43) and adjustment: original (obs.) vs equated (fair.). It also shows score correlations with criterion measures. With the full dataset, the original score Obs.Avge correlates $r=.99$ with the equated FairMAvge. We considered FairMAvge as a benchmark because it contains all data and it is adjusted for eventual differences in rater's leniencyseverity. Considering the condition of $67 \%$ of the full data set, FairMAvge correlates $r=.93$ with FairMAvge_67, which is slightly above its correlation $(r=.90)$ with original 
Obs.Avg_67. Considering the $43 \%$ of full data set condition, FairMAvge correlates $r=.74$ with FairMAvge_43 and Obs.Avg_43.

Looking to criterion validity, we observe that the pattern of correlations with full dataset of creativity and external measures (intelligence and personality) remained the same if we calculate creativity scores that preserved up to $67 \%$ of data. With $43 \%$ of the full data set, the magnitude of the correlations tended to be slightly lower. The only exception occurred when we examine the correlation of creativity measures with the personality dimension of openness to experience, which tended to have the same magnitude of correlations across all scores we calculated regardless of amount of missing data. We didn't observe differences in validity coefficients when comparing equated versus original scores where rater's leniency-severity is not controlled for.

\section{Discussion}

In this reanalysis, we explored descriptive statistics of model parameters to see how well MFRM fits the data and if raters and tasks differ substantially in their severity/difficulty. We also introduced missing data to check if subjects' adjusted scores would be more similar to full dataset scores and also if subjects' adjusted scores exhibit the same correlation with external variables (validity check).

MFRM produced good fit to the data according to the usual fit statistics benchmarks employed in Rasch analysis (Linacre, 2013). We found that the tasks' $\beta$ 's difficulty parameters didn't differ substantially. Raters $\alpha$ 's, on the other hand, were more dispersed. One rater was substantially less severe $(\alpha=-1.18)$ than the other two, who had quite similar $\alpha$ 's parameter ( $\alpha$ 's =.54 and .64), resulting a difference of -1.72 and 1.82 logits (-1.18 to .54 and -1.18 to .64$)$. Seeing this difference in perspective, the difficulty of receiving score 1 vs 0 was $\tau_{1}=-1.01,2$ vs 1 was $\tau_{2}=-.14$, and 3 vs 2 was $\tau_{3}=1.15$. Thus, about 1.1 logits imply a raw score change of 1 point. Therefore, the difference between the 
lenient rater and the other two can be greater than one score point.

It is important to emphasize the utility of construct maps, a byproduct of the application of MFRM, to visualize all parameters of the model on a common metric. This enhances the understanding of the latent variable metric, making it less arbitrary and more meaningful (Embretson, 2006; Primi, 2014b; Wyse, 2013). It is interesting to note that the distribution of creative potential as measured by metaphor tasks is positively skewed. For instance, when we see theta distributions $(\theta)$ in relation to the actual response categories thresholds $(\tau)$ we learn that most subjects' ideas receive an overall score as "not at all creative". This indicates that that creative idea generation is quite a difficult task. It was also observed on another study with similar tasks (Primi, 2014a). Besides the adjustment of scores with respect to individual rater's differences, the use of MFRM can be valuable tool in the applied field of creativity measurement. Construct maps and task and raters parameters (difficulty and consistency) can be a valuable tool to understand raters' behavior in terms of difficulty-leniency as well as reliability. Task parameters can shed light to their usefulness and consistency. Finally, category thresholds maps and parameters can assist in the development of scoring rubrics. Overall, MFRM is a valuable tool that can potentially improve the quality of creativity assessment.

Despite differences among raters, we found that the subjects' unadjusted scores correlated very strongly with adjusted scores. These differences were not high enough to bias subject's measures. Differences in adjusted and unadjusted scores are expected to exist when responses are scored by a combination of two raters, for instance, and that different raters pairings have marked differences in leniency-severity dimension. This was not the case for the present dataset. Since only one rater differed from other two, the three possible pairings of two raters will have relatively similar levels of severity.

With respect to the comparison of scores based on partial versus complete data, we 
found that when we had $67 \%$ of rater data preserved to score each idea (33\% of missing data), the subjects' scores are pretty similar to the ones calculated with the full dataset.

Correlations between scores calculated with the full dataset with those calculated with partial data ranged from .90 to .93 . When we have $43 \%$ of rater's data preserved (57\% of missing data) the correlations ranged from .73 to .74 . The correlation of adjusted scores with adjusted full dataset ones tended to be little better than unadjusted scores (.92 vs .90 and .74 vs .73). Nevertheless, these differences are very small. In general, these results indicate that you can have very similar scores with about $1 / 3$ workload reduction on raters.

With respect to validity data we found that correlations of creativity with external variables (fluid intelligence and big five domain scales) were very similar in their patterns and magnitudes between scores calculated with the full dataset versus a partial data setparticularly when we have $2 / 3$ of ratings available. It is noticeable that for some key constructs like openness and $g f$ (measured by Raven's progressive matrices), the adjusted scores seem to do a better job in keeping the magnitude of validity coefficients when compared with non-adjusted scores: Openness .20 vs .17, Ravens .32 vs 30 . These results suggest that, when using different rater pairs, accounting for rater differences with MFRM seems to be paying off in terms of validity of the measures.

In summary, it is not clear that unadjusted scores for rater differences or non-equated scores will do poorly as compared to adjusted/equated scores, although this may have occurred because of the absence of big differences in the difficulty of the pseudo booklets composed of rater pairs. We turn now to a second reanalysis where we have more raters and a bigger sample that will enable us to investigate in depth the effects of adjustments on similarity of convergence of scores with full dataset, reliability, and validity.

\section{Study 2: Ratings for Alternate Uses and Instances Tasks}

\section{Method}


This study is a reanalysis of data from a study of divergent thinking (Jauk et al., 2013, 2014). Participants generated responses to six divergent thinking tasks that were rated holistically for creativity by four raters on a scale of 1 to 4 . The primary goal in this reanalysis is to fit a MFRM model, to compare the reliability and validity of original scores versus adjusted (i.e., equated) scores that are corrected for rater differences, and to do so under two conditions of simulated missing data that reflect different patterns of rater assignments.

\section{Participants}

The sample of this study included 298 healthy, German-speaking individuals (101 male) aged between 18 and 55 years $(M=30, S D=10.68)$. Participants were recruited via a local newspaper and took part in a larger research project on intelligence, creativity, and personality (cf. Jauk et al., 2013, 2014). Participants were paid for taking part in the study.

\section{Measures}

Divergent thinking tasks. Participants completed six divergent thinking (DT) tasks, which are common measures of creative potential. The DT tasks included three alternate uses (AU) and three instances (IN) tasks. In the alternate uses tasks, participants were required to find many uncommon and creative uses for a can, a knife, and a hairdryer. In the IN tasks, participants were asked to figure out many novel and uncommon solutions to the problems What can make noise?, What can be elastic?, and What can be used for faster locomotion?. All tasks were administered on a standard PC. Participants worked on each task for two minutes and entered their ideas via a keyboard.

All ideas produced in the divergent thinking tasks were evaluated for creativity. For each task, we first compiled alphabetically-sorted lists of all non-redundant ideas. Each idea was then evaluated by four experienced raters on a 4-point scale ranging from 0 (not creative) to 3 (very creative). Raters were instructed that a creative idea is both novel/unusual and 
useful/clever.

Creative activities and achievement. Creative activities and achievement were assessed using the Inventory of Creative Activities and Achievement (ICAA; Diedrich et al., in press). The ICAA comprises two distinct scales for the assessment of creative activities (i.e., personal, everyday creativity) and publicly recognized achievement across eight domains. Reliability, construct, and criterion validity of the ICAA were previously examined (Diedrich et al., in press). In the ICAA, everyday creative activities are assessed using six items for each of the eight domains such as wrote a short literary work (e.g., poem, short story) or wrote a blog entry. Participants indicate on a five-point Likert-type scale how often they carried out these activities within the past 10 years (never, one to two times, three to five times, 5-10 times, and more than 10 times). Creative achievement is assessed on an ordinal checklist that asks for the maximal attained achievement in each domain (i.e., I have never been engaged in this domain [0 points] to I have already sold some of my work in this domain [10 points]). The achievement scale is conceptually similar to the Creative Achievement Questionnaire (CAQ; Carson et al., 2005) and was shown to have good convergent validity (Jauk et al., 2014). Additionally, participants were asked to write down the most creative achievements of their life to account for potential achievements that were not included in the ICAA. All achievements were rated for creativity by five independent raters on a six-point rating scale ranging from 0 (not creative) to 5 (highly creative). The average rating of the five most creative achievements was used as an additional score of rated creative achievement.

Openness to experience. A central personality correlate of creativity, openness to experience was assessed using an established German Big Five measure (Big Five Structure Inventory [BFSI]; Arendasy et al., 2011). The test is based on Item Response Theory (IRT) and was shown to have good reliability and validity (Arendasy et al., 2011).

Intelligence. Intelligence was assessed using the Intelligence Structure Battery 
(INSBAT; Arendasy et al., 2004). The INSBAT is an IRT-based computerized test grounded in the Cattell-Horn-Carroll model of intellectual ability (for an overview, see McGrew, 2009) and allows for tailored testing. Target reliability was set to $\alpha=.60$, which results in an average of 10 items per test, or an average duration of 10 minutes per test.

\section{Design and Statistical Modeling}

The DT data encompasses the responses of 298 people to six DT tasks. The average number of responses to one DT task was $12(S D=6.13)$, and the total number of responses to all 6 tasks was 22,064 . The total number of non-redundant responses that were evaluated by the raters was $9,013(45 \%)$. The final total data set comprising all responses with ratings from four raters hence included 88,256 data points.

To evaluate the effects of missing data, we computed average creativity scores based on complete data and compared them to scores computed with incomplete data (50\% and $25 \%$ ). For the complete data set there were on average 74 responses per subject (across six DT tasks), with a minimum of 19 and maximum of 144 . Since each response was scored by four raters there were, on average, 296 data points per subject to calculate the average creativity scores. For incomplete data scores, we employed a Balanced Incomplete Block (BIB) design. For 50\%-BIB we restricted the data to a subset of two out of four raters (six possible pairwise combinations), and subsets were equally varied between participants. For 25\%-BIB we further restricted data to 3 out of 6 DT tasks within participants (we used 10 subsets of 3 tasks). So, the $50 \%$ group had ratings from combinations of 2 of the 4 raters, but for all 6 tasks. The $25 \%$ group has ratings from combination of 2 of the 4 raters, but for only 3 tasks. Appendix A has detailed information on the combinations of raters and tasks used.

The BIB was an essential part of this design to guarantee the connectedness of the dataset, defined as when "a network of links exists through which every element that is involved in producing an observation is directly or indirectly connected to every other 
element of the same assessment context" (Eckes, 2009, p. 39). With this connectedness we can estimate all the parameters of the model—abilities, item difficulties, and rater leniency vs severity - in the same metric. This topic is related to linking-equating in the Item Response Theory family of techniques (Cook \& Eignor, 1991; Wolfe, 1999). BIB is an ideal design to achieve this condition of complete linkage because it ensures an optimally balanced level of connectedness.

Once the relevant datasets were established, we computed parameters of the model using Formula 1 and scores of average creativity using Formula 3. The calibration/estimation of the model was calculated on the software FACETS (Linacre, 2013), which employs the joint maximum-likelihood estimation method (JMLE). We ran models three times: the benchmark with complete data, then with $50 \%$ of the data, and finally with $25 \%$ of the data. We compared reliability and validity indices across these two levels of incompleteness.

\section{Results}

First, we present some general information on tasks and raters calculated with the full dataset. The upper part of Table 3 shows average scores on each task, the fair average - the observed score corrected from other elements on the facets—-beta parameters of task difficulty (and their standard errors), fit statistics (infit and oufit), and item-theta (latent score) correlations. Fit indexes are directly proportional to residuals, that is, the difference between observed versus expected scores. Values below 1.4 usually indicate acceptable fit (Linacre, 2013). The middle part of Table 1 shows the same information but now for the raters. The lower part of Table 3 shows classical inter-rater reliability measures based on absolute agreement (consensus measures): the percent agreement and the weighted Kappa index.

\section{Insert Table 3 here}

Examining Table 3 we can observe, first, that fit indices were acceptable since none of the infit and outfit indices were 1.4 or higher. Second, the task and rater parameters vary 
systematically. $\alpha_{j}$ varies from -.44 (can) to 1.04 (fast locomotion), and $\beta_{i}$ varies from -.41

(J2) to $.49(\mathrm{~J} 1)$, a difference of .90 logits. The ratio of the raters' alpha parameters differences between any two raters divided by their standard errors would reach statistical significance. This signals a potential problem of confounding subject abilities with rater characteristics if we disregard rater facets when dealing with an incomplete dataset.

Third, the kappa consensus measures of inter-rater reliability indicate fair to moderate agreement. If part of the inter-rater disagreements is due to differences in levels of severity, the index should increase when we recalculate kappa with relaxed criteria for agreement. So we recalculated indices allowing that a difference of one point will still be considered agreement. Indeed, the numbers in parenthesis in the lower part of Table 1 shows that interrater reliability goes up when we consider a more liberal criterion. Absolute agreement reaches levels higher than $90 \%$, and weighted kappas are now in in the range of fair to substantial agreement (in kappa calculations we supplied the following weights for the perfect to worst disagreement: 1 for a distance of 0,1 for a distance of 2,4 for a distance of 3 , 8 for a distance of 4 ). This result again reinforces the point that raters vary in their leniency. If we want to reduce raters' burden and use subsets of raters to score each idea and we don't make any effort to control rater effects, we will potentially have cofounded scores in the incomplete datasets.

But to what extent do rater differences impact scores? Does MFRM correct for the potential differences that may arise? We present a second group of results comparing subjects' creativity scores in the full dataset with the two conditions of incomplete data. We calculated six scores for each subject. Three scores were based on raw average of ratings across raters, tasks, and responses with the full data (Obs.Avge_00), with half raters 50\% (Obs.Avge_50), and half raters and half tasks 25\% (Obs.Avge_25). We also calculated adjusted average scores according to the MFRM model controlling for raters and task 
differences again in these three conditions: full (FairAvge_00), 50\% (FairAvge_50), and 25\% (FairAvge_25).

In the full dataset we had an average of 296 points of data per subject (subject's $n$ responses on 6 tasks rated by 4 raters) comprising a total of $N=88,256$ observations. In the $50 \%$ dataset we had an average of 144 points of data per subject comprising a total of $N=$ 44,128 observations. And in the $25 \%$ dataset we had an average of 70 points of data per subject comprising a total of $N=21,890$ observations. Table 4 presents descriptive statistics and inter-correlations of these six measures.

\section{Insert Table 4 here}

Examining Table 4 we see that for the full dataset, unadjusted (ObsAvge_00) and adjusted scores (FairAvge_00) are practically the same, $r=.99$. This result is expected since every rater scores all subjects in every task and therefore there is no confounding of rater/tasks with subjects. This constitutes the best-case scenario where we observed all data points and used adjusted scores.

With incomplete data we see a different picture. The worst-case scenario occurs when we compare adjusted full dataset scores (FairAvge_00) with unadjusted 25\%-dataset (ObsAvge_25). In this case the correlation drops to $r=.63$. This constitutes a lower benchmark of examining the effect of reducing data points - and not adjusting scores of combinations of raters and tasks that vary in levels of difficulty/severity. This contrast suggests that lower numbers of observations and, consequently, different pairings of raters that vary in their level of severity, introduces substantial error and reduces the correlation with the full dataset.

One important question is what happens when we adjust scores for raters and tasks effects using MFRM? These adjustments control rater effects only. Therefore, any improvement to the worst-case scenario will be an estimate of improvement when the 
measurement model accounts for the raters' different levels of severity.

The correlation of the adjusted full dataset score (FairAvge_00) with adjusted 25\%dataset scores (FairAvge_25) increases to $r=.74$ (as compared with $r=.63$ discussed above). Interestingly, we observe the same effect of the model adjustments with the 50\%-dataset. Adjusted full dataset scores (FairAvge_00) correlates $r=.79$ with unadjusted 50\%-dataset scores (ObsAvge_50). But it correlates $r=.94$ with adjusted 50\%-dataset scores (FairAvge_50). It is not a surprise to see that correlations increase if we have more data. But interestingly, when using MFRM to adjust scores, we can practically obtain similar results with using half of the data. Confirming our expectations, scores derived from incomplete data do well when we adjust scores for raters' severity and tasks' difficulty.

Figure 2 illustrates the effects of incomplete data and score adjustments. It shows four scatterplots where points represent subjects' scores comparing benchmark scores with scores calculated with incomplete data. Y-axis shows the reference benchmark adjusted scores with full dataset. $\mathrm{X}$-axis of the four figures shows scores calculated under four conditions: adjusted 25\%-dataset (upper-left), unadjusted 25\%-dataset (upper-right), adjusted 50\%dataset (lower-left), unadjusted 50\%-dataset (lower-right). The different shades and shape of the points indicate the six possible pairwise combinations of raters scoring the ideas (the six booklets). We also show the six regression lines predicting benchmark scores from incomplete data conditioned on particular pairwise combinations of raters.

It can be seen that in the figures of the right column - the unadjusted scores - that the regression lines have different intercepts, suggesting that different combinations of raters map on different levels on the benchmark scores. In the figures of the left column-the adjusted scores — we see that these differences in intercept practically disappear, especially on the 50\%-dataset where we have more data points. These figures show clearly that the improvements in approximating incomplete data scores to the benchmark scores is due to 
correction of varying levels of severity that follows when we combine different pairings of raters.

\section{Insert Figure 2 here}

Another important question concerns reliability. How reliable are a subject's latent scores with incomplete data? In IRT models, reliability coefficients vary as function of latent ability because the particular mix of items may measure specific sectors of the latent scale better than others. In Figure 3 we show the information function plotting reliability coefficients (Y-axis) as a function of the latent scale (X-axis). We rescaled results of the information function to the standard reliability metric of 0 to 1 . Each point represents a subject. We calculated reliability three times: (a) full data-set (black), (b) 50\%-dataset (dark gray), and (c) 25\%-dataset (light gray). We plotted smoothed average lines of points in these three conditions.

\section{Insert Figure 3 here}

Again, the full-dataset group is a benchmark to compare the other two conditions of incomplete data. Median reliabilities were good: full-dataset .90 ( $75 \%$ of points above .87$)$, $50 \%$-dataset .80 ( $75 \%$ of points above .77$)$, and $25 \%$-dataset .75 ( $75 \%$ of points above .70$)$. Since the full dataset contains an abundant number of data points per subject $(M=296)$ estimates of latent reliability are very high. It is noticeable that with half the data points we still reach high reliability. With one quarter of the data points the reliability is lower but doesn't deteriorate past acceptable levels.

The final question concerns criterion validity. To what extent is test-criterion validity compromised when using shorter tests (fewer tasks and raters)? Do rater effects compromise validity? Does MFRM correct an eventual decrease in criterion validity when adjusting scores for differences in rater severity? Table 5 presents correlations of unadjusted versus adjusted scores on three conditions of completeness on columns with criterion measures on 
rows (Originality, Fluency, IQ, Openness to experience, ICAA's Creative Activities, ICAA's Creative Achievement and Rated Creative Achievement).

\section{Insert Table 5 here}

As expected, validity coefficients are lower when we have fewer data points. The more marked decrease occurs when we have a quarter of the original data points. But the decrease is higher for unadjusted scores as compared with adjusted scores. Specifically, with adjusted scores with $50 \%$ of data, the decrease in validity coefficients is minimal. In general, MFRM correction helps to maintain the criterion validity in a manner that is unaffected by potential bias of differences in raters' severity.

\section{Discussion}

In this second study, we further explored reliability and validity of unadjusted and adjusted scores in conditions of missing data, simulating a situation of less burden on the work of raters. The present dataset was composed of extensive samples of creativity production from the subjects (a mean of 74 ideas per subject) in six tasks scored by four raters. Therefore, in the partial data conditions, while simulating "booklets" of pairs of raters and triplets of tasks, we were in a better position to find more variability in these conditions. For instance, we had 6 booklets combining unique rater's pairings in one condition and 60 booklets combining unique combination of rater pairings and task triples.

We found a similar pattern of raters' $\alpha$ parameters variability as was found in Study 1 (the more demanding rater was .9 logits apart from the more lenient rater). We also found a substantial variability of tasks' $\beta$ difficulty parameters (the most difficult task was 1.48 logits apart from the easiest one). Therefore, this result indicates that there can be a lot of variability in terms of difficulty of the particular combination of 
raters and tasks in each booklet. The main question of this study was to what extent the adjustment of MFRM could help decrease the potential negative impact on reliability and validity of using less information when we reduce rater burden?

We found clear evidence that MRMP adjustment helped to maintain validity and reliability. First, when we use half of the data available, the correlations of subjects' unadjusted partial scores with full dataset scores was $r=.79$, while the correlation with the adjusted scores was practically unchanged $r=.94$. When we use one quarter of the data available these correlations were $r=.64$ for the unadjusted and $r=.74$ for the adjusted scores. This clearly shows that part of the lack of convergence between scores is due to differences in raters biasing subjects' scores. Second, criterion validity was systematically higher for adjusted scores compared with unadjusted scores. When using half of the data available, the criterion validity coefficients were very similar to the coefficients calculated with full dataset. Finally, the reliability coefficients were in the good range even if we use half of the data available.

\section{General Discussion}

Creativity assessment with divergent thinking tasks relies heavily on scoring the quality of people's ideas. This creates a complex measurement structure involving persons, tasks (and ideas within tasks), and raters. Most studies, however, don't model

possible systematic differences among raters. The present study examines the impact of individual rater differences in the reliability and validity of creativity assessments, and it explains how an MFRM approach can model and correct for these differences.

In summary, the use of MFRM reveals systematic differences among raters that will bias subjects' creativity scores-i.e., become errors of measurement of the subject facets-if not accounted for. Studies 1 and 2 showed that differences in leniency versus 
severity between raters and differences in task difficulty can be quite pronounced. There was a difference in beta of more than 1 logit in study 2 and a difference of more than 1 in alpha in study 1 , which represents substantial variations given the tight scale. Nevertheless, there was virtually no difference between observed and adjusted measures when using the full data, that is, when all raters scores all ideas. On the other hand, rater individual differences were influential when using incomplete data because combinations of raters will co-occur with particular subsets of subjects. As shown in Study 2, individual rater differences, that is, rater's systematic errors of measurement, were confounded with subjects' scores. In this context, MRFM proved to be an efficient method to control for rater differences and adjust scores, thus removing this systematic error, making scores more convergent, and maintaining validity when compared with complete data.

The benefits of MFRM become particularly apparent when using incomplete data designs. Removing $50 \%$ of data (Study 2) substantially affects reliability and validity evidence for unadjusted scores, whereas adjusted scores appear largely unaffected. For example, correlations of the unadjusted score with the benchmark score (adjusted score based on complete data) is only .76 and correlations with other creativity measures are markedly reduced, suggesting a lack of criterion validity. In contrast, the adjusted score is still very highly correlated with the benchmark (.94) and therefore correlations with other creativity measures are also unchanged. This effect is similar but less pronounced for designs with 33\% missing data (Study 1). However, when introducing missing data beyond 50\% (Study 1: 43\%, Study 2: 75\%) deviations from benchmark scores are substantial. 
The lack of convergence between benchmark scores and incomplete data scores could be due to two main reasons: (a) a general effect of a reduced sample of behavior, like a test with reduced number of items, that always reduces reliability and, consequently, validity (Primi, 2012), and (b) raters' systematic errors of measurement confounded with subjects' scores. Our findings suggest that adjustments for variability in rater and task facets by means of MFRM, that is, the second factor, compensates fairly well for the loss due to incomplete data up to a certain extent of roughly $50 \%$ missing data. This suggests that the lack of convergence in this case is mostly due to systematic error in raters than to the reduction of the sampled behavior. However, when data become too sparse, they don't reach the benchmarks of the full dataset scores, although we still see an improvement with MFRM adjustments. This suggests that there exists a cutoff point related to the amount of information that is needed for a given level of reliability. After that, reducing sampled behavior available to assess creativity of a given person becomes a more influential factor in reducing reliability and validity.

A related topic reinforcing the advantage of MFRM is related to the quantification of reliability. In divergent thinking tasks, we usually have a sample of a subject's behavior produced after prompted by a particular task. If we consider each idea as an "item", since we have a variable number of ideas per subject, we have a situation as if subjects had answered different tests composed by a variable number of items. Then we have raters who score each idea. The usual way to quantify reliability is to calculate some sort of inter-rater reliability (Stemler, 2004). This method quantifies only one facet of this complex design, namely, how similar the scores of two independent raters of a given idea are. But the final quantity of interest is the subject's estimated creativity score that is based in a large amount of data points from the aggregation of rater, task, 
and response facets. MFRM uses the general IRT concept of information function (Ayala, 2009) to compute model-based reliabilities for all elements within facets. Thus, MFRM makes it possible to quantify the reliability of each element within each facet using all the information available. Therefore, MFRM provides a more efficient way to estimate reliability in creativity assessment.

In Study 2, for instance, we found the interrater reliability ranged from fair to moderate (Kappa indices ranged from .17 to .42). But we found that Rasch subjects' creativity scores reliability were excellent, around .90. Although the inter-rater reliability was in the lower range it should be clear that it informs the reliability of only one given idea. For each subject, we have several ideas. Therefore, we have an extended sample of behavior, based on aggregation of rater scores, to estimate a person's creative ability. Because of that MFRM yields higher reliability estimates as compared to the reliability of raters alone. In summary MFRM offers a more comprehensive account for quantifying reliability.

In Study 2 the estimation of reliability via MFRM shed light on the fact that the implemented design (six tasks X four raters) produced an abundant amount of information to estimate each subject's creativity score, achieving very high reliability. Therefore, we conclude that there is room for reducing raters' workload-a serious issue in subjective scoring (Forthmann et al., 2017)—without losing psychometric information if MFRM is used to adjust rater's differences. But of course, there are limits to this method. Our findings suggest that adjusted scores work well for incomplete data designs with missing data up to $50 \%$. This figure will translate to the following simple (rough) rules: (a) with 100 data points per subject from $n$ responses X 6 tasks X 2 raters we would predict an average reliability of .70 (given that we have kappa interrater 
agreement around .25) and (b) with 150 data points would achieve an average reliability of .80 .

In summary, we conclude that MFRM can be considered a powerful means to reduce rater load in creativity research while helping to maintain reliability and validity that would otherwise be compromised. It also highlights an important problem of linking-equating where we need to link raters to a common metric to have creativity measurements on a comparable scale when different subjects are scored by different raters. 


\section{References}

Amabile, T. M. (1982). Social psychology of creativity: A consensual assessment technique. Journal of Personality and Social Psychology, 43, 997-1013.

Arendasy, M., Sommer, M., \& Feldhammer, M. (2011). Manual Big-Five Structure Inventory. BFSI. Mödling: Schuhfried Gmbh.

Arendasy, M., Sommer, M., \& Feldhammer, M. (2011). Manual Big-Five Structure Inventory. BFSI. Mödling: Schuhfried Gmbh.

Arendasy, M., Hornke, L. F., Sommer, M., Häusler, J., Wagner-Menghin, M., Gittler, G., Wenzl, M. (2004). Manual Intelligence-Structure-Battery. INSBAT. Mödling: SchuhfriedGmbh.

Ayala, R. J. de (2009). The theory and practice of Item Response Theory. New York: Guilford Press

Benedek, M., Jauk, E., Kerschenbauer, K., Anderwald, R., \& Grond, L. (2017). Creating art: An experience sampling study in the domain of moving image art. Psychology of Aesthetics, Creativity, and the Arts, 11, 325-334. doi: 10.1037/aca0000102

Benedek, M., Mühlmann, C., Jauk, E., \& Neubauer, A. C. (2013). Assessment of divergent thinking by means of the subjective top-scoring method: Effects of the number of topideas and time-on-task on reliability and validity. Psychology of Aesthetics, Creativity, and the Arts, 7, 341-349.

Carson, S. H., Peterson, J. B., \& Higgins, D. M. (2005). Reliability, validity, and factor structure of the creative achievement questionnaire. Creativity Research Journal, 17, $37-50$.

Christensen, P. R., Guilford, J. P., \& Wilson, R. C. (1957). Relations of creative responses to working time and instructions. Journal of Experimental Psychology, 53, 82-88.

Costa, P. T., Jr., \& McCrae, R. R. (1992). Revised NEO Personality Inventory (NEO-PI-R) 
and NEO Five-Factor Inventory (NEO-FFI) professional manual. Odessa, FL: Psychological Assessment Resources.

Cook, L. L., \& Eignor, D. R. (1991). IRT equating methods. Educational measurement: Issues and practice, 10(3), 37-45.

Cronbach, L. J., Gleser, G. C., Nanda, H., \& Rajaratnam, N. (1972). The dependability of behavioral measurements: Theory of generalizability for scores and profiles. New York, NY: Wiley.

Cropley, D. H., \& Kaufman, J. C. (2012). Measuring functional creativity: Non - expert raters and the Creative Solution Diagnosis Scale. The Journal of Creative Behavior, 46, 119-137.

Diedrich, J., Benedek, M., Jauk, E., \& Neubauer, A.C. (2015). Are creative ideas novel and useful? Psychology of Aesthetics, Creativity and the Arts, 9, 35-40. doi:10.1037/a0038688

Diedrich, J., Jauk, E., Silvia, P.J., Gredlein, J.M., Neubauer, A.C., \& Benedek, M. (in press). Assessment of real-life creativity: The Inventory of Creative Activities and Achievements (ICAA). Psychology of Aesthetics, Creativity, and the Arts.

Eckes, T. (2009). Many-facet Rasch measurement. In S. Takala (Ed.), Reference supplement to the manual for relating language examinations to the Common European Framework of Reference for Languages: Learning, teaching, assessment (Section H). Strasbourg, France: Council of Europe/Language Policy Division.

Embretson, S. E. (2006). The continued search for nonarbitrary metrics in psychology. American Psychologist, 61, 50 -55. doi:10.1037/0003-066X .61.1.50

Forthmann, B., Holling, H., Zandi, N., Gerwig, A., Çelik, P., Storme, M., \& Lubart, T. (2017). Missing creativity: The effect of cognitive workload on rater (dis-) agreement in subjective divergent-thinking scores. Thinking Skills and Creativity, 23, 129-139. 
Gamer, G., Lemon, J., Fellows I., Singh, P. (2015). Various Coefficients Interrater

Reliability and Agreement. R package version 0.84 URL http://cran.rproject.org/web/packages/irr/.

Hekkert, P., \& van Wieringen, P. C. W. (1996). Beauty in the eye of expert and nonexpert beholders: A study in the appraisal of art. American Journal of Psychology, 109, 389407.

Jauk, E., Benedek., M., Dunst, B., \& Neubauer, A. C. (2013). The relationship between intelligence and creativity: New support for the threshold hypothesis by means of empirical breakpoint detection. Intelligence, 41, 212-221.

Jauk, E., Benedek, M., \& Neubauer, A. C. (2014). The road to creative achievement: A latent variable model of ability and personality predictors. European Journal of Personality, $28,95-105$.

Kaufman, J. C., Baer, J., Cropley, D. H., Reiter-Palmon, R., Sinnett, S. (2013). Furious activity vs. understanding: How much expertise is needed to evaluate creative work? Psychology of Aesthetics, Creativity, and the Arts, 7, 332-340.

Kaufman, J. C., Lee, J., Baer, J., \& Lee, S. (2007). Captions, consistency, creativity, and the consensual assessment technique: New evidence of reliability. Thinking Skills and Creativity, 2, 96-106.

Kaufman, J. C., Plucker, J. A., \& Baer, J. (2008). Essentials of creativity assessment. Hoboken, NJ: Wiley.

Kozbelt, A. (2005). Factors affecting aesthetic success and improvement in creativity: A case study of the musical genres of Mozart. Psychology of Music, 33, 235-255.

Linacre J. M. (2004). KR-20 or Rasch reliability: Which tells the "truth"? Rasch Measurement Transactions, 11, 580-581.

Linacre, J. M. (1994). Many-Facet Rasch Measurement. Chicago: Mesa Press. 
Linacre, J. M. (2013) Facets computer program for many-facet Rasch measurement, version 3.71.2. Beaverton, Oregon: Winsteps.com

Long, H., \& Pang, W. (2015). Rater effects in creativity assessment: A mixed methods investigation. Thinking Skills and Creativity, 15, 13-25.

MacKinnon, D. (1962). The nature and nurture of creative talent. American Psychologist, 17, 484-495.

McGrew, K. (2009). CHC theory and the human cognitive abilities project: Standing on the shoulders of the giants of psychometric intelligence research. Intelligence, 37, 1-10.

Nusbaum, E. C., Silvia, P. J., \& Beaty, R. E. (2017). Ha ha? Assessing individual differences in humor production ability. Psychology of Aesthetics, Arts, and Creativity, 11, 231241.

Primi, R. (2014a). Divergent productions of metaphors: Combining many-facet Rasch measurement and cognitive psychology in the assessment of creativity. Psychology of Aesthetics, Creativity, and the Arts, 8(4), 461.

Primi, R. (2014b). Developing a fluid intelligence scale through a combination of Rasch modeling and cognitive psychology. Psychological assessment, 26(3), 774.

Primi, R. (2012). Psicometria: fundamentos matemáticos da Teoria Clássica dos Testes. [Psychometrics: mathematical foundations of classical test theory]. Avaliação Psicológica, 11(2), 297-307. http://pepsic.bvsalud.org/scielo.php?script=sci_arttext\&pid=S167704712012000200015\&lng=pt\&tlng=pt.

Ruthsatz, J., Detterman, D., Griscom, W. S., \& Cirullo, B. A. (2008). Becoming an expert in the musical domain: It takes more than just practice. Intelligence, 36, 330-338.

R Core Team (2016). R: A language and environment for statistical computing. R Foundation for Statistical Computing, Vienna, Austria. URL https://www.R-project.org/. 
Sawyer, R. K. (2006). Explaining creativity: The science of human innovation. New York, NY: Oxford University Press.

Silvia, P. J., \& Beaty, R. E. (2012). Making creative metaphors: The importance of fluid intelligence for creative thought. Intelligence, 40, 343-351.

Silvia, P. J., Winterstein, B. B., Willse, J. T., Barona, C. M., Cram, J. T., Hess, K. I., ... Richard, C. A. (2008). Assessing creativity with divergent thinking tasks: Exploring the reliability and validity of new subjective scoring methods. Psychology of Aesthetics, Creativity, and the Arts, 2, 68-85.

Stemler, S. E. (2004). A comparison of consensus, consistency, and measurement approaches to estimating interrater reliability. Practical Assessment, Research \& Evaluation, 9(4). Retrieved August 15, 2014 from http://PAREonline.net/getvn.asp?v=9\&n=4

Wolfe, E. W. (1999). Equating and item banking with the Rasch model. Journal of Applied Measurement, 1(4), 409-434.

Wright B. D. \& Masters G. N. (1982). Rating scale analysis. Chicago: MESA.

Wyse, A. E. (2013). Construct maps as a foundation for standard setting. Measurement: Interdisciplinary Research and Perspectives, 11(4), 139-170. 
Table 1. Basic statistics and psychometric information on metaphor tasks and raters

\begin{tabular}{|c|c|c|c|c|c|c|c|}
\hline \multicolumn{8}{|c|}{ Tasks parameters and fit statistics } \\
\hline & Average & Fair & & & & & \\
\hline & Score & $\begin{array}{c}\text { Average } \\
\text { Score }\end{array}$ & $\beta_{i}$ & $\begin{array}{c}\mathrm{SE} \text { of } \\
\beta_{i},\end{array}$ & infit & outfit & $\begin{array}{c}\mathrm{r}_{\mathrm{rt}} \\
\text { (Meas) }\end{array}$ \\
\hline 1 boredom & 1.64 & 1.56 & -0.20 & 0.08 & .92 & 0.81 & 0.70 \\
\hline 2 disgust & 1.50 & 1.39 & 0.20 & 0.09 & 1.11 & 1.18 & 0.58 \\
\hline
\end{tabular}

Raters parameters and fit statistics

\begin{tabular}{rrrrrrrr}
\hline & $\begin{array}{c}\text { Average } \\
\text { Score }\end{array}$ & $\begin{array}{c}\text { Fair } \\
\text { Average } \\
\text { Score }\end{array}$ & \multicolumn{1}{c}{$\begin{array}{c}\text { SE of } \\
\alpha_{j}\end{array}$} & \multicolumn{1}{c}{ infit } & \multicolumn{1}{c}{ outfit } & \multicolumn{1}{c}{$\begin{array}{c}\mathrm{r}_{\mathrm{rt}} \\
\text { (Meas) }\end{array}$} \\
\hline $\mathrm{J} 1$ & 1.34 & 1.29 & .54 & .12 & 1.03 & .97 & .50 \\
$\mathrm{~J} 2$ & 1.32 & 1.26 & .64 & .12 & 1.19 & 1.13 & .46 \\
$\mathrm{~J} 3$ & 2.05 & 2.16 & -1.18 & .09 & .91 & .89 & .69
\end{tabular}

Raters reliability measures: percent agreements (below diagonal) and weighted kappa (above diagonal) indexes. In parenthesis the same indices with a more lenient criteria for agreement.

\begin{tabular}{lrrr} 
& \multicolumn{1}{c}{1} & \multicolumn{1}{c}{2} & \multicolumn{1}{c}{3} \\
\cline { 2 - 4 } 1 J1 & & & .30 \\
& & $.26(.30)$ & $(.39)$ \\
2. J2 & 68.7 & & .19 \\
& $(92.0)$ & & $(.25)$ \\
3. J3 & 41.6 & 38.9 & \\
& $(80.5)$ & $(77.1)$ & \\
\hline
\end{tabular}

Note: Fair Average Score is calculated as demonstrated in Equation 3 considering the rater facet. $\beta_{i}$, task $i$ difficulty parameter, $\alpha_{j}$ rater $j$ parameter. $\mathrm{r}_{\mathrm{rt}}$ (Meas) is the correlation of item scores rater total scores that combine other items or rater score. In the lower part of the matrix presented at the bottom of the table we show inter-rater reliability index calculated from standard formula and, in parenthesis, the results considering a more lenient criterion for agreements tolerating a difference of one point. In the calculation of Weighted kappa we supplied the following weights for the perfect to worst disagreement: 1 for a distance of 0,1 for a distance of 2, 4 for a distance of 3, 8 for a distance of 4 . All inter-rater reliability calculations made on R package irr (Gamer, Lemon, Fellows, \& Singh, 2015). 
Table 2. Correlations of scores under various conditions of completeness and adjustment with criterion measures.

\begin{tabular}{|c|c|c|c|c|c|c|c|c|c|c|}
\hline Vars & M & SD & $\min$ & $\max$ & $\begin{array}{l}\infty \\
\infty \\
\sum_{0} \\
0 \\
0\end{array}$ & 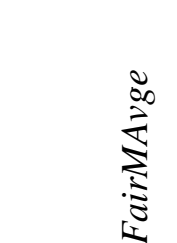 & 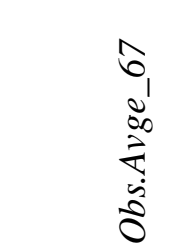 & 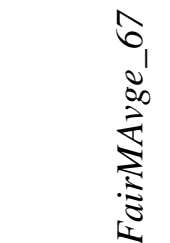 & $\begin{array}{l}3 \\
\forall \\
0 \\
\infty \\
\sum_{1}^{\infty} \\
\dot{0} \\
0\end{array}$ & 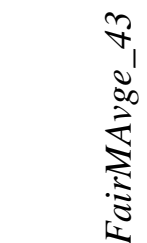 \\
\hline \multicolumn{11}{|l|}{$\begin{array}{l}\text { Creativity } \\
\text { measures }\end{array}$} \\
\hline Obs.Avge & 1.6 & 0.5 & 1.0 & 3.2 & & & & & & \\
\hline FairMAvge & 1.5 & 0.5 & 1.0 & 3.3 & $0.994 * * *$ & & & & & \\
\hline Obs.Avge_67 & 1.6 & 0.5 & 1.0 & 3.3 & $0.906 * * *$ & $0.903 * * *$ & & & & \\
\hline FairMAvge_67 & 1.5 & 0.5 & 1.0 & 3.4 & $0.923 * * *$ & $0.932 * * *$ & $0.976 * * *$ & & & \\
\hline Obs.Avge_43 & 1.6 & 0.6 & 1.0 & 3.0 & $0.743 * * *$ & $0.737 * * *$ & $0.404 * * *$ & $0.475^{* * *} *$ & & \\
\hline \multicolumn{11}{|l|}{$\begin{array}{l}\text { Intelligence } \\
\text { measures }\end{array}$} \\
\hline cf_series & 7.9 & 1.6 & 3.0 & 11.0 & 0.149 & 0.147 & 0.145 & 0.146 & 0.082 & 0.095 \\
\hline cf_matrix & 6.4 & 1.1 & 3.0 & 9.0 & -0.004 & -0.003 & -0.024 & -0.023 & 0.036 & 0.041 \\
\hline letter_sets & 8.8 & 2.5 & 3.0 & 14.0 & 0.152 & 0.144 & 0.129 & 0.122 & 0.127 & 0.138 \\
\hline paper_folding & 5.3 & 2.3 & 1.0 & 10.0 & $0.211 *$ & $0.207 *$ & $0.211^{*}$ & $0.221 *$ & 0.143 & 0.134 \\
\hline ravens & 10.0 & 3.3 & 0.0 & 16.0 & $0.320 * * *$ & $0.323 * * *$ & $0.308 * * *$ & $0.323 * * *$ & $0.181 *$ & $0.177 *$ \\
\hline number_series & 8.1 & 2.0 & 4.0 & 14.0 & 0.169 & 0.157 & 0.154 & 0.12 & 0.126 & 0.167 \\
\hline \multicolumn{11}{|l|}{$\begin{array}{l}\text { Personality } \\
\text { measures }\end{array}$} \\
\hline n_ffi & 2.8 & 0.7 & 1.0 & 4.6 & 0.126 & 0.12 & 0.142 & 0.14 & 0.055 & 0.051 \\
\hline e_ffi & 3.6 & 0.5 & 1.8 & 4.7 & $-0.178^{*}$ & $-0.190 *$ & $-0.188^{*}$ & $-0.228 * *$ & -0.092 & -0.033 \\
\hline o_ffi & 3.4 & 0.5 & 2.2 & 4.7 & $0.236 * *$ & $0.251 * *$ & $0.177 *$ & $0.203 *$ & $0.233 * *$ & $0.254 * *$ \\
\hline a_ffi & 3.5 & 0.5 & 2.2 & 4.6 & -0.006 & -0.002 & 0.008 & -0.004 & -0.002 & 0.055 \\
\hline c_ffi & 3.5 & 0.5 & 2.0 & 4.9 & $-0.218 *$ & $-0.209 *$ & $-0.237 * *$ & $-0.215^{*}$ & -0.089 & -0.104 \\
\hline
\end{tabular}


Table 3. Basic statistics and psychometric information on tasks and raters

\begin{tabular}{|c|c|c|c|c|c|c|c|}
\hline sks paramet & $\begin{array}{c}\text { cs and fit s } \\
\text { Average } \\
\text { Score }\end{array}$ & $\begin{array}{c}\text { atistics } \\
\text { Fair } \\
\text { Average } \\
\text { Score }\end{array}$ & $\beta_{i}$, & $\begin{array}{c}\text { SE of } \\
\beta_{i},\end{array}$ & infit & outfit & $\begin{array}{c}\mathrm{r}_{\mathrm{rt}} \\
\text { (Meas) }\end{array}$ \\
\hline 1 can & 1.01 & 1.00 & -0.44 & 0.02 & 0.68 & 0.69 & 0.34 \\
\hline 2 knife & 0.77 & 0.76 & 0.23 & 0.02 & 1.28 & 1.27 & 0.32 \\
\hline 3 hairdryer & 0.89 & 0.88 & -0.13 & 0.02 & 1.10 & 1.09 & 0.28 \\
\hline 4 noise & 0.96 & 0.96 & -0.33 & 0.02 & 0.69 & 0.69 & 0.23 \\
\hline 5 elastic & 0.98 & 0.97 & -0.37 & 0.02 & 1.27 & 1.24 & 0.27 \\
\hline 6 fast loc. & 0.51 & 0.50 & 1.04 & 0.02 & 1.15 & 1.14 & 0.23 \\
\hline
\end{tabular}

Raters parameters and fit statistics

\begin{tabular}{cccccccc}
\hline & $\begin{array}{c}\text { Average } \\
\text { Score }\end{array}$ & $\begin{array}{c}\text { Fair } \\
\text { Average } \\
\text { Score }\end{array}$ & \multicolumn{1}{c}{$\alpha_{j}$} & \multicolumn{1}{c}{$\begin{array}{c}\text { SE of } \\
\alpha_{j}\end{array}$} & infit & outfit & $\begin{array}{c}\mathrm{r}_{\mathrm{rt}} \\
\text { (Meas) }\end{array}$ \\
\hline J1 & 0.62 & 0.63 & 0.49 & 0.01 & 1.31 & 1.29 & 0.36 \\
J2 & 0.95 & 0.97 & -0.41 & 0.01 & 1.04 & 1.07 & 0.22 \\
J3 & 0.89 & 0.91 & -0.27 & 0.01 & 0.76 & 0.76 & 0.14 \\
J4 & 0.72 & 0.74 & 0.19 & 0.01 & 0.94 & 0.94 & 0.24
\end{tabular}

Raters reliability measures: percent agreements (below diagonal) and weighted kappa (above diagonal) indexes. In parenthesis are the same indices with more lenient criteria for agreement.

\begin{tabular}{lrrrr} 
& \multicolumn{1}{c}{1} & \multicolumn{1}{c}{2} & \multicolumn{1}{c}{3} & \multicolumn{1}{c}{4} \\
\cline { 2 - 5 } 1 J1 & & & .24 & .28 \\
& & $.21(.29)$ & $(.41)$ & $(.55)$ \\
2. J2 & 47.3 & & .17 & .42 \\
& $(91.4)$ & & $(.42)$ & $(.66)$ \\
3. J3 & 49.5 & 51.5 & & .22 \\
& $(95.4)$ & $(96.0)$ & & $(.60)$ \\
4. J4 & 51.1 & 60.4 & 54.0 & \\
& $(96.3)$ & $(97.0)$ & $(97.9)$ & \\
\hline
\end{tabular}

Note: Fair Average Score is calculated as demonstrated in Equation 3 considering the rater facet. $\beta_{i}$, task $i$ difficulty parameter, $\alpha_{j}$ rater $j$ parameter. $\mathrm{r}_{\mathrm{rt}}$ (Meas) is the correlation of item scores rater total scores that combine other items or rater score. In the lower part we present inter-rater reliability index calculated from standard formula and, in parenthesis, the results considering a more lenient criteria for agreements tolerating a difference of one point. In the calculation of Weighted kappa we supplied the following weights for the perfect to worst disagreement: 1 for a distance of 0,1 for a distance of 2, 4 for a distance of 3,8 for a distance of 4. All inter-rater reliability calculations made on R package irr (Gamer, Lemon, Fellows Singh, 2015). 
Table 4. General descriptive statistics and intercorrelations among the six original observed average ratings of ideas (Obs) versus corrected averages by the MFRM (Fair). On the diagonal we present a global index of reliability calculated from standard errors of the latent scores that we estimated for each subject.

\begin{tabular}{lrllllllllr}
\hline Variable & Mean & SD & $\min$ & $\max$ & 1 & 2 & 3 & 4 & 5 \\
\hline 1. ObsAvge_00 & 0.79 & 0.11 & 0.43 & 1.12 & $\mathbf{0 . 8 9}$ & & & & \\
2. ObsAvge_50 & 0.79 & 0.14 & 0.43 & 1.16 & 0.79 & $\mathbf{0 . 7 9}$ & & & \\
3. ObsAvge_25 & 0.80 & 0.17 & 0.39 & 1.27 & 0.64 & 0.79 & $\mathbf{0 . 7 2}$ & & \\
4.FairAvge_00 & 0.81 & 0.11 & 0.42 & 1.12 & 0.99 & 0.79 & 0.63 & ----- & \\
5. FairAvge_50 & 0.81 & 0.11 & 0.40 & 1.20 & 0.94 & 0.83 & 0.66 & 0.95 & ---- \\
6. FairAvge_25 & 0.81 & 0.14 & 0.39 & 1.29 & 0.74 & 0.64 & 0.85 & 0.74 & 0.78 \\
\hline
\end{tabular}


Table 5. Correlations of scores under various conditions of completeness and adjustment with criterion measures.

\begin{tabular}{|c|c|c|c|c|c|c|c|c|c|c|c|c|}
\hline Criterion & \begin{tabular}{l}
8 \\
8 \\
8 \\
8 \\
\multirow{4}{*}{} \\
$\dot{0}$ \\
0
\end{tabular} & 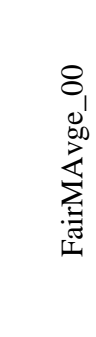 & 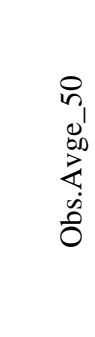 & 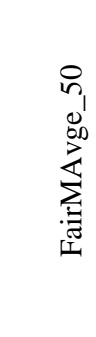 & 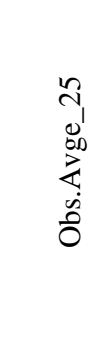 & 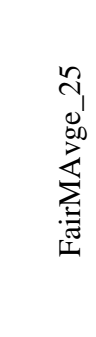 & 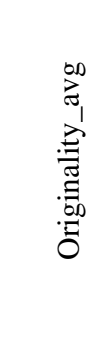 & 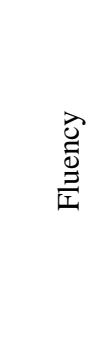 & 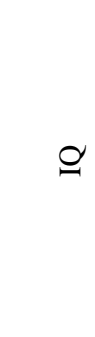 & 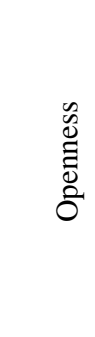 & 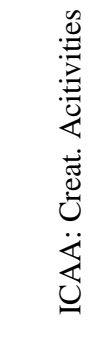 & 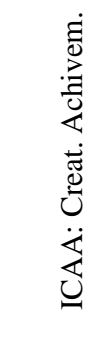 \\
\hline Originality_avg & $.974^{* * *}$ & $.981^{* * *}$ & $.762^{* *}$ & $.936^{* *}$ & $.628^{* *}$ & $.750^{* *}$ & & & & & & \\
\hline Fluency & .058 & .068 & .051 & .061 & .082 & .074 & .021 & & & & & \\
\hline IQ & $.341^{* *}$ & $.359^{* *}$ & $.243^{* *}$ & $.295^{* *}$ & $.197^{* *}$ & $.215^{* *}$ & $.352^{* *}$ & $.217^{* *}$ & & & & \\
\hline Openness & $.160^{* *}$ & $.154^{* *}$ & .065 & $.132^{*}$ & .026 & .072 & $.139^{*}$ & $.269^{* *}$ & $.137^{*}$ & & & \\
\hline ICAA: Creat. Acitivities & $.224^{* *}$ & $.216^{* *}$ & $.128^{*}$ & $.180^{* *}$ & .066 & .106 & $.199^{* *}$ & $.322^{* *}$ & $.144^{*}$ & $.494^{* *}$ & & \\
\hline ICAA: Creat. Achivem. & $.219^{* *}$ & $.212^{* *}$ & $.131^{*}$ & $.187^{* *}$ & $.126^{*}$ & $.167^{* *}$ & $.208^{* *}$ & $.281^{* *}$ & $.281^{* * *}$ & $.368^{* *}$ & $.656^{* *}$ & \\
\hline $\begin{array}{l}\text { Peer-Rated Creat. } \\
\text { Achievem. }\end{array}$ & $.366^{* *}$ & $.369^{* *}$ & $.283^{* *}$ & $.343^{* *}$ & $.217^{* *}$ & $.259^{* *}$ & $.362^{* *}$ & $.144^{*}$ & $.311^{* * *}$ & $.210^{* *}$ & $.336^{* *}$ & $.401^{* *}$ \\
\hline
\end{tabular}



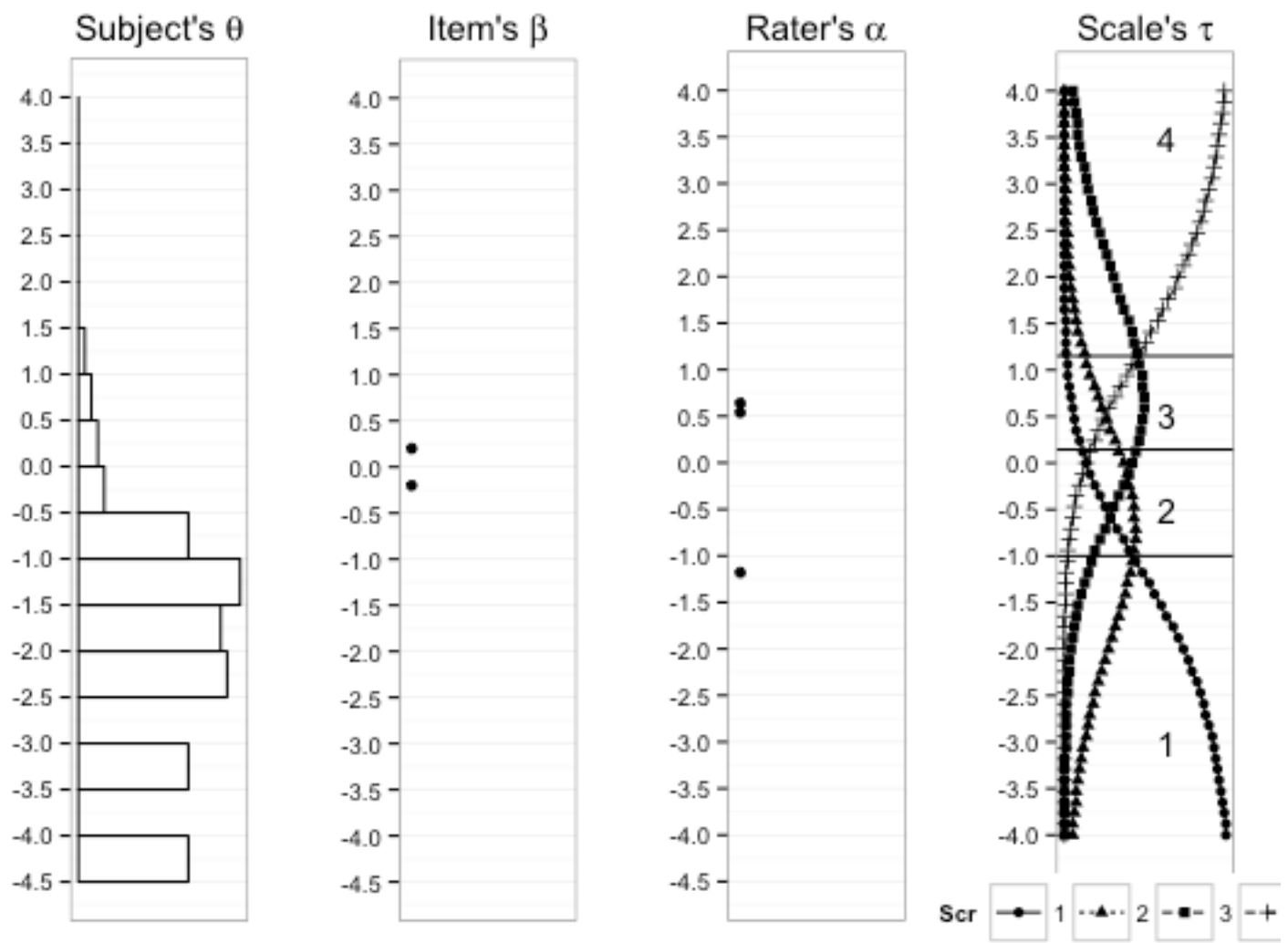

Figure 1. Construct map showing the distributions of subject's abilities, item's difficulties, rater's severities and category thresholds. 


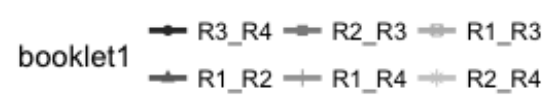

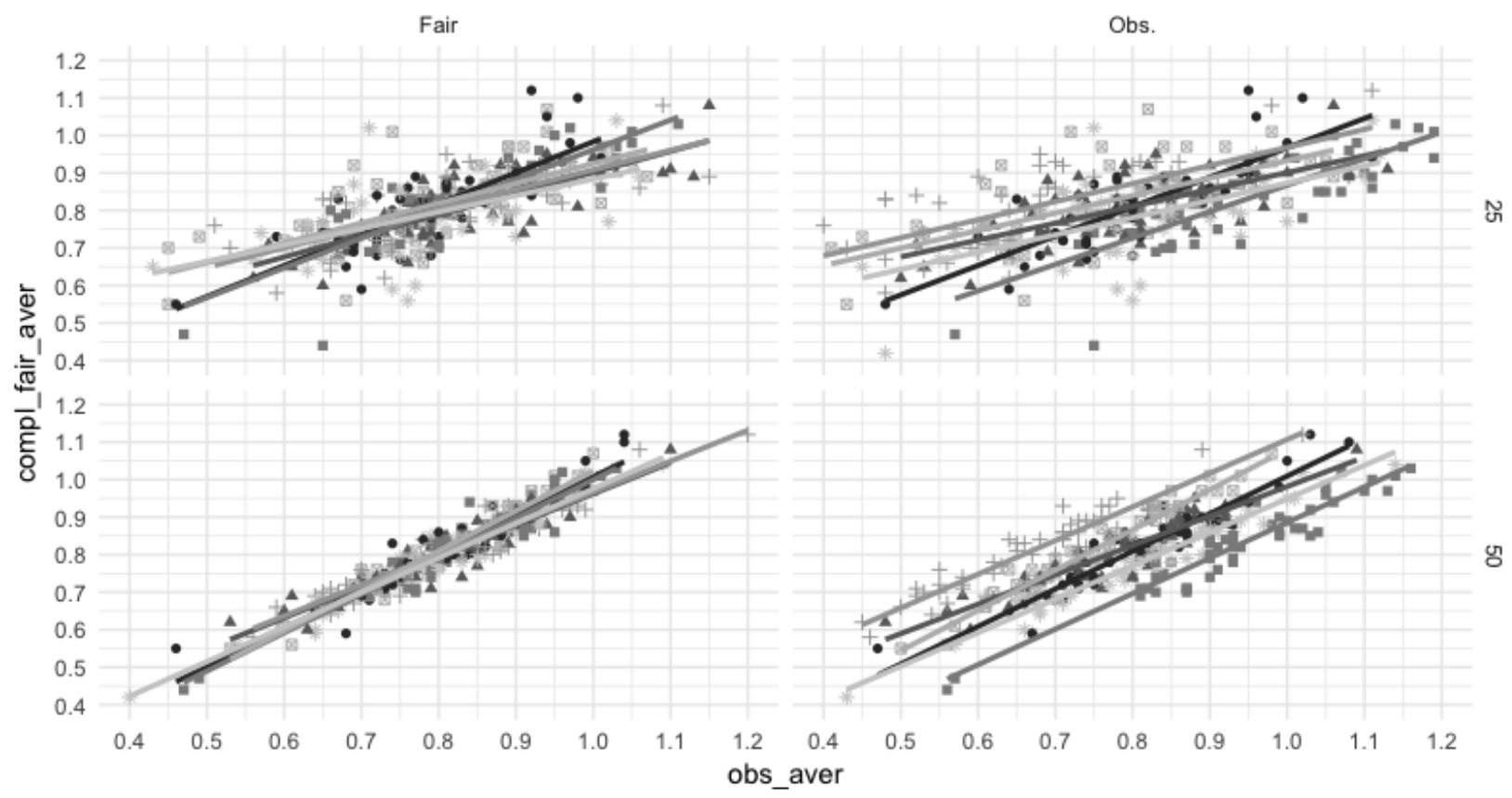

Figure 2. Comparing benchmark scores (adjusted score with full dataset; Y-axis) with scores in four conditions of completeness and adjustment: adjusted 25\%-dataset (upper-left), unadjusted 25\%-dataset (upper-right), adjusted 50\%-dataset (lower-left), unadjusted 50\%dataset (lower-right). 


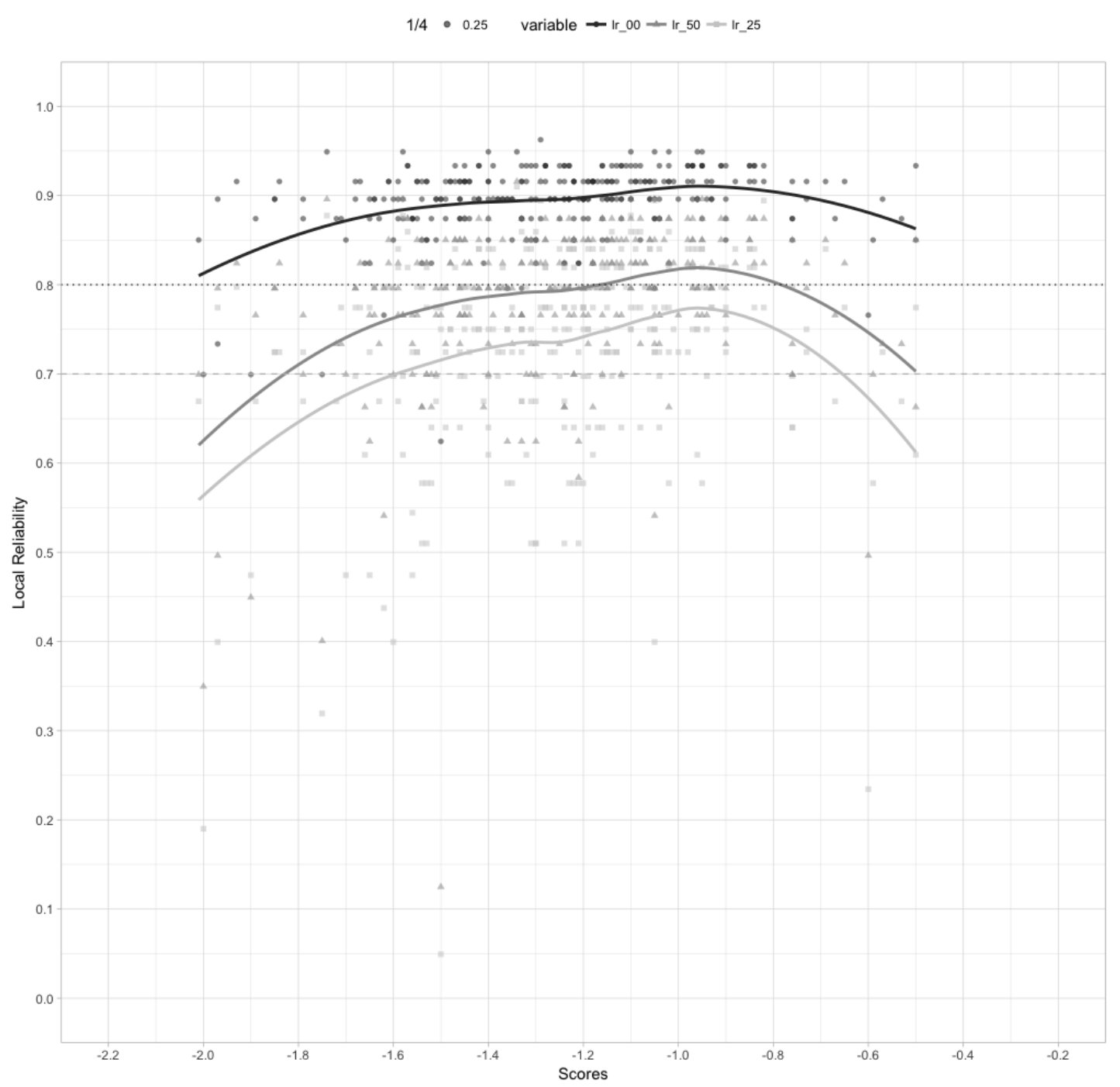

Figure 3. Local reliability of latent scores under three conditions of completeness (black: full data-set, dark-gray: 50\%-dataset and light-gray: 25\%-dataset) 
Appendix A. The balanced incomplete block design combinations of raters and tasks

$$
50 \% \text {-BIB }
$$

Considering six raters we have six combinations that we call booklets. In each booklet each rater is combined with each other. In the Table A1 each row corresponds to booklet and each cell contains the number of the particular rater on the booklet. Each subject were randomly assigned to one of these six booklets, that is, to one of these combinations of two raters.

Table A1. Booklets 1. Six (booklets) combinations of 2 out of four raters

[1] [2]

[1] 34

[2] 12

[3] $22 \quad 3$

[4] 14

[5] 13

[6] 24

\section{$25 \%$-BIB}

We further construed in the same way ten combinations of three out of six tasks. In Table A2 each row corresponds to a booklet in which three out of six tasks are combined. Now each pairwise combination of any two tasks appear repeated in two booklets (for instance tasks 3 and 5 appear in booklets 1 and 2, tasks 5 and 6 in booklets 1 and 7 and so on).

Table A2. Booklets 2. Ten (booklets) combinations of three out of six DT-tasks 
[1] [2] [3]

[1] $3 \quad 5 \quad 6$

[2] $23 \quad 3 \quad 5$

[3] $1313 \quad 4$

[4] $20 \begin{array}{lll}2 & 4 & 6\end{array}$

[5] $14 \quad 4 \quad 5$

[6] $122 \quad 6$

[7] $4 \quad 5 \quad 6$

[8] 13130

[9] $20 \begin{array}{lll}2 & 3 & 4\end{array}$

[10] $12 \quad 2 \quad 5$

BIB 25 were formed combining Booklets 1 and 2 resulting in 60 booklets. This is formed from 6 (combinations of raters) times 10 combinations (combinations of tasks). Therefore in $25 \% \mathrm{BIB}$ each subject were randomly assigned to one of these combinations. Therefore in this BIB each subject will be scored by two raters on only three tasks. 


\section{Tutorial: How to run Many Facet Rasch Models in the analysis of creativity ratings}

\section{Goal}

The purpose of this tutorial is to show how to run Many Facet Rasch Model analysis for a typical dataset where raters evaluate subjects' responses in a set of divergent thinking tasks. We will show how to run the analysis on FACETS (http://www.winsteps.com/facets.htm), a commercial software that is a benchmark for running these models, as well as how to run the same analysis on a the open-source R package TAM (REF?). We start by showing how to setup the data for both analyses using $R$.

\section{Facets}

\section{Reshape the original dataset to run the analysis on FACETS}

Usually datasets are organized in a wide format, that is, observations in lines and variables in columns. Suppose you have a file stored in Excel called $d f . x L s x$. You can read it into $\mathrm{R}$ using the package readxl. If you don't have the package installed yet, install it first.

$$
\text { install.packages("readxl") }
$$

Then activate the package and read the file into a data frame called $d f$. The first column has a subject identifier. The other six columns have scores for three raters (rater1 to rater 3 ) scoring two metaphor tasks (met1 and met2). This data comes from Silvia \& Beaty (2012) used in Study 1. The data file can be downloaded from here

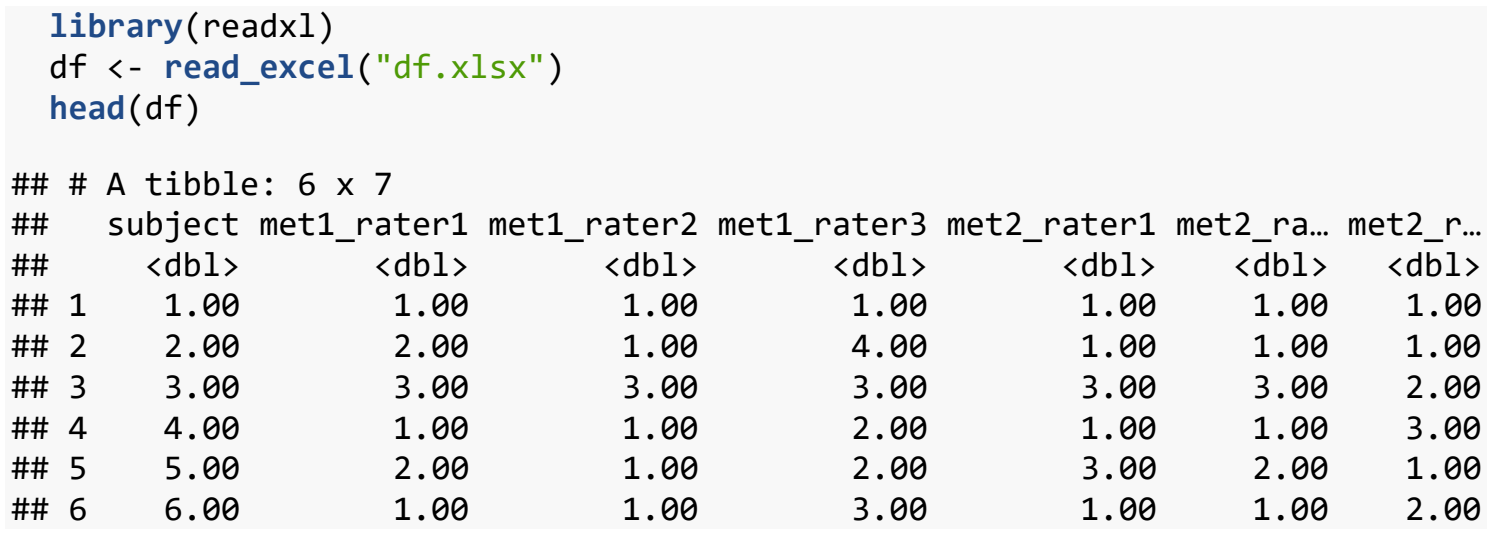

In order to run FACETS, we need to transform these data into a long format, that will have four columns: subject, task, rater and score: (a) subject records the subject identifier, (b) task records the task number, (c) rater records the rater number, and (e) score records the rating a rater has given to a particular subject on a particular task. So each row will contain data for a combination of a subject answering a task and its score given by a particular rater. In order to reshape the $d f$ file we will use the function gather from the package tidyverse. See Wickham \& Grolemund (2017) for more details. First you install this package if you haven't already done so. 
Then we activate the package and use the function gather to reshape $d f$. Gather will transform a data frame into long format. It will get the six columns and stack them into one column. We need two variables: (a) one variable to keep the information about which original column was being stacked. This variable will record the original variable names; (b) another variable will have the values of the original column. You define the name of first new variable using the gather's function key argument and the name of second new variable using the value argument. Note that our code uses pipe operator \% \% that passes the left-hand side object/variable as the first argument of the right-side function. This makes the code more easily readable.

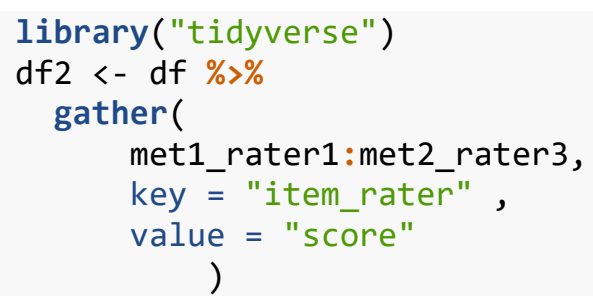

Note that now column names met1_rater1, met1_rater2, met1_rater3, met2_rater1, met2_rater2, met2_rater3 are values of a new variable called item_rater and score containing the ratings. Now we have 804 rows representing 134 subjects times six itemrater combinations. Note that the information of rater and task is recorded in the values of var. We need to extract this information and create two new variables. We will use a dplyr's separate function to do this. We pass as argument the names of the new variables that will be produced while splitting the original variable item_rater:

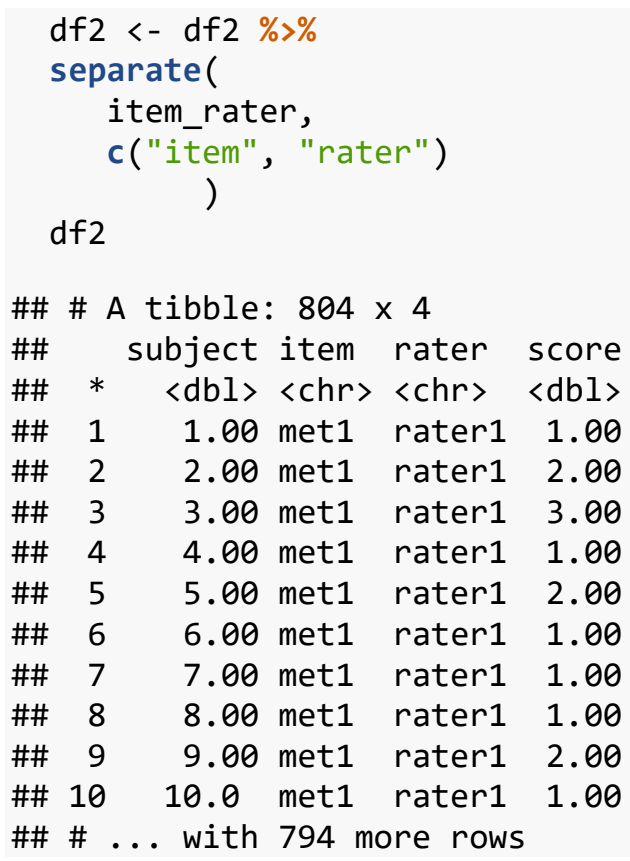

We now recode rater and task as integers, which makes it easier to handle them in FACETS.

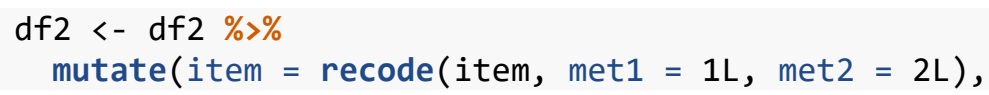




$$
\text { ) }
$$

$$
\text { rater }=\operatorname{recode}(\text { rater, } \text { rater } 1=1 \mathrm{~L}, \text { rater } 2=2 \mathrm{~L} \text {, rater } 3=3 \mathrm{~L})
$$

\section{Save the data file}

Now we have the file ready for the FACETS program to read. We can finally export this file

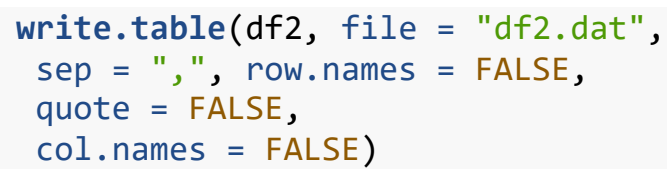

Once the datafile is ready we need to prepare the commands to run FACETS. It is out of the scope of this tutorial to present a detailed analysis of FACETS syntax. The interested reader finds an excellent tutorial written by Mike Linacre, the author of the program here [https://winsteps.com/facetman/tutorialpdfs.htm]. Below we present the command lines to run FACETS with the data we just exported. We commented each line of code informing briefly what they do. You can copy and paste them to a plain text file and then run them in FACETS. But before that substitute the comment starter character \# by ;

\section{Example of FACETS file including code and data}

\# This is Facet code not $R$ code

\# When preparing the final file substitute the comment character "\#" by ";"

\# Title for the analysis

Title = Silvia \& Beaty (2012) Creativity Ratings

\# Number of facets: Subjects, task and raters

Facets $=3$

\# Specifies type of data in each column of data related to each facet. The tree $1 \mathrm{~N}$ , $2 N, 3 N$ instructs that each of the three columns are numbers " $N$ "

Delements $=1 \mathrm{~N}, 2 \mathrm{~N}, 3 \mathrm{~N}$

\# Specifies the model. The first three ?,?,? indicate that each subject can intera ct with each task and each rater. The $R 4$ specifies to run a rating scale model wit $h$ four response options.

Model = ?, ?, ?, R4

\# Instructs FACETS that subjects facet (facet number 1) is the only one positively oriented. This means that high scores are associated to high ability and conseque ntly higher likelihood of high item scores. Task and rater facets are negatively o riented, that is, the higher their score the more difficult the tasks and the more strict the raters are. Therefore, in more difficult items and stricter raters it is more likely to observe lower item scores.

Positive $=1$

\# This specifies which facets are not to be centered. Since we specified 1 (Subjec ts) the latent scores of subjects will not be constrained to have a mean of zero.

The other two facets - tasks and raters - will have this constraint, that is, thei $r$ mean will be set to zero.

Noncenter $=1$

\# Specifies what facets are the rater facets so FACETS can calculate inter-rater a greement

Inter-rater $=3$ 


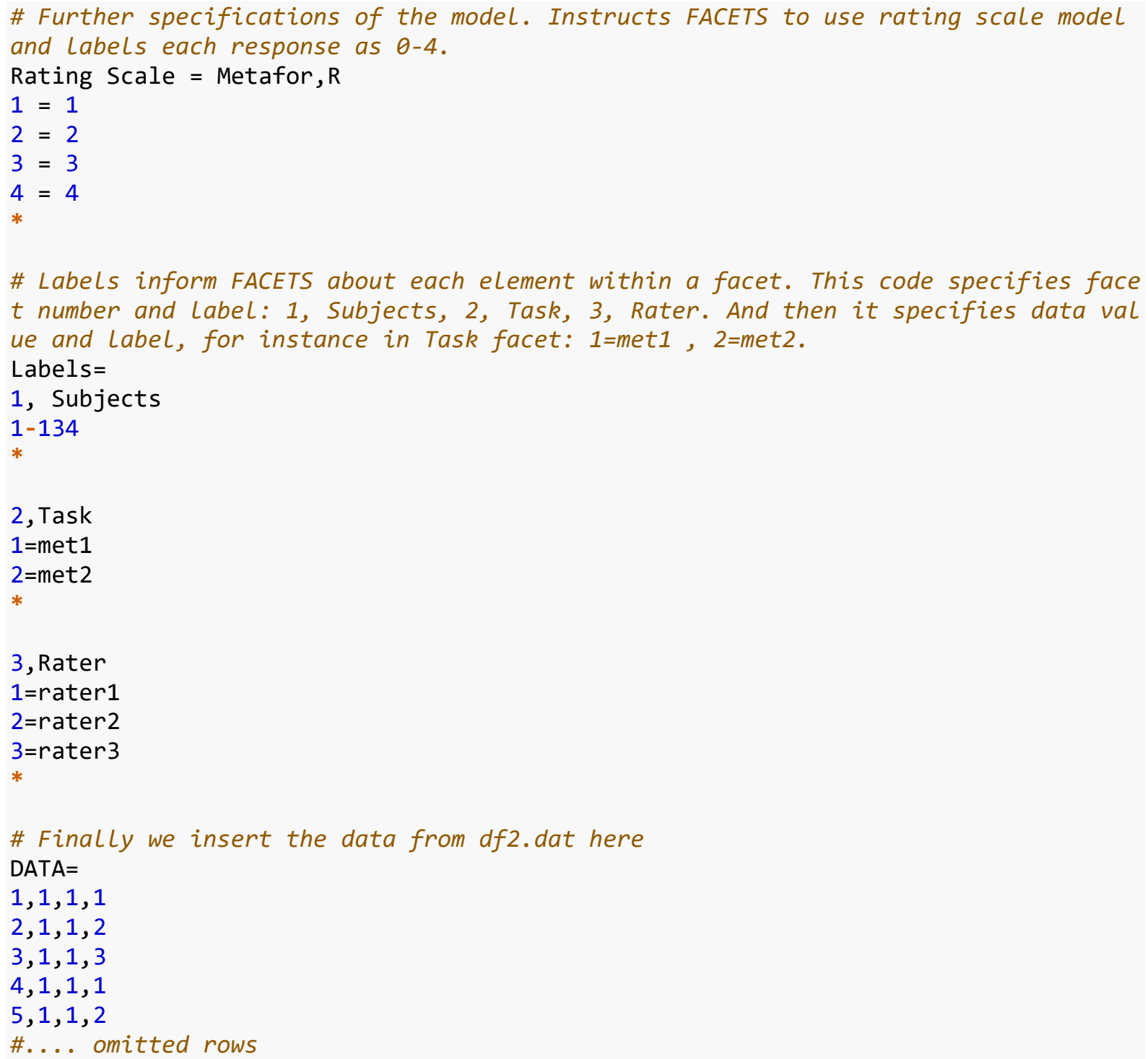

The full commands can be downloaded from this link:

[http://www.labape.com.br/metaphor/df2 cmd.dat] and the FACET output from this link: [http://www.labape.com.br/metaphor/DF2 CMD.out.txt].

Running facets with $\mathrm{R}$ package TAM

Alexander Robitzsch [https://github.com/alexanderrobitzsch] created two packages TAM and immer that can run several types of facet model. TAM has a function tam. mmL.mfr that mimics FACETS using joint maximum likelihood estimation method. (see: Robitzsch \& Steinfeld 2018; Robitzsch \& Steinfeld, 2017; Robitzsch, Kiefer, \& Wu, 2018). We will run the analysis using TAM.

First you need to install TAM in case you haven't done yet.

$$
\text { install.packages("TAM") }
$$


Then we will prepare the datafile to be analyzed by TAM. It requires slightly different format having subjects+raters in rows and tasks in columns. We will reshape $d f 2$ and save it into $d f 3$ with this specific shape. Note we first transform the variable score so it shifts from 1 to 4 to 0 to 3 . Starting from 0 is more consistent with the parametrization used in TAM. Then we use a function from tidyr called spread that does the opposite of gather. It takes the values of the variable item that are scattered over rows and spreads them into columns. We need to indicate which variable contains the values scattered in rows as key argument: key = item and which column contains values as a value argument: value = score. This will transpose items into columns. Then the column names will be its values 1 and 2 . It is more convenient to have column names as characters. So we rename column names.

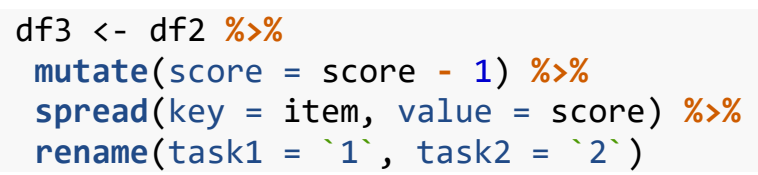

We now can activate TAM and run the analysis. Below we run the analysis and store the results in an list object called $f i t$. We can investigate elements of $f i t$ using the function names ( $f i t$ ). We then call a function summary passing $f i t$ as its argument to show the main results.

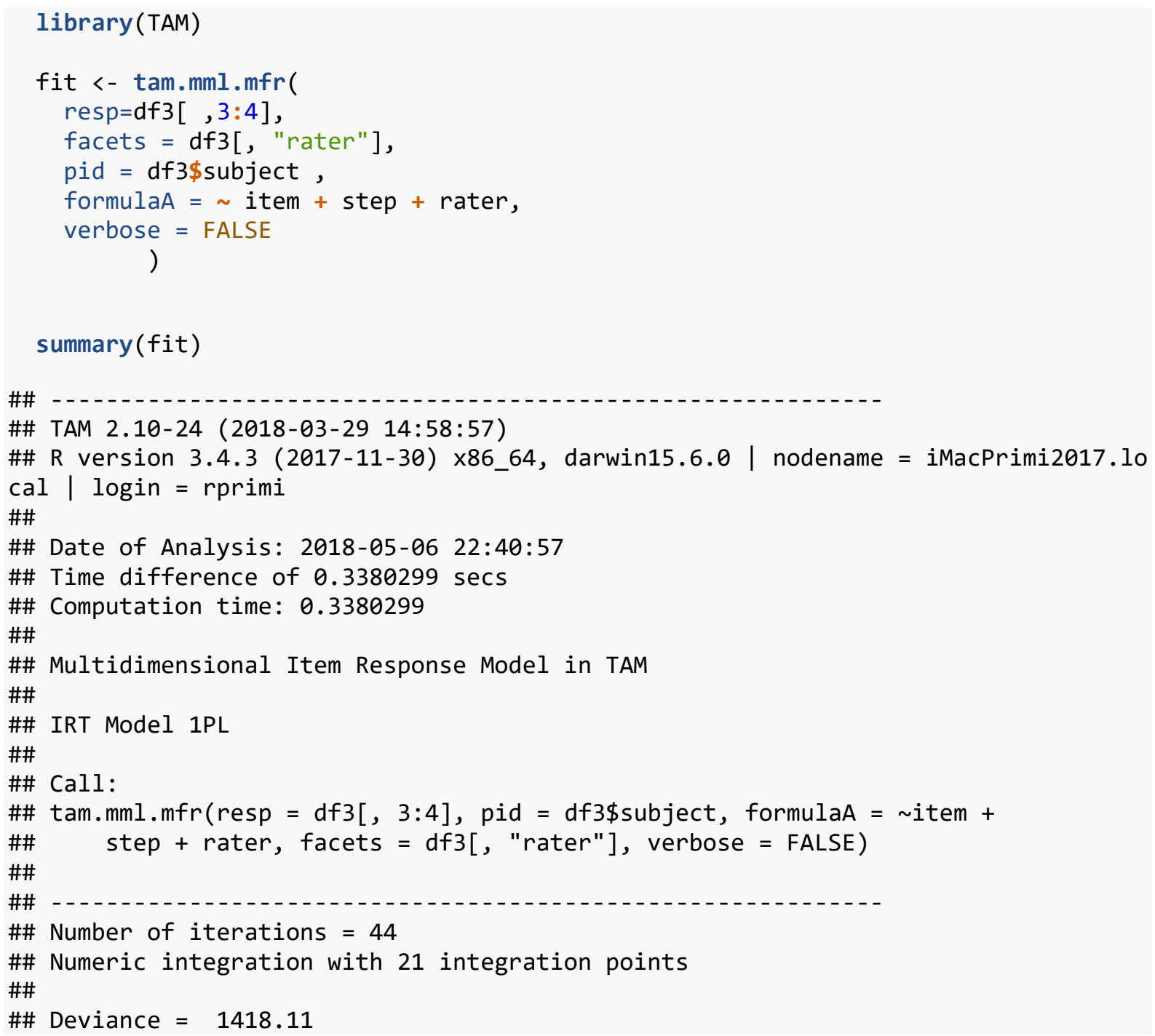




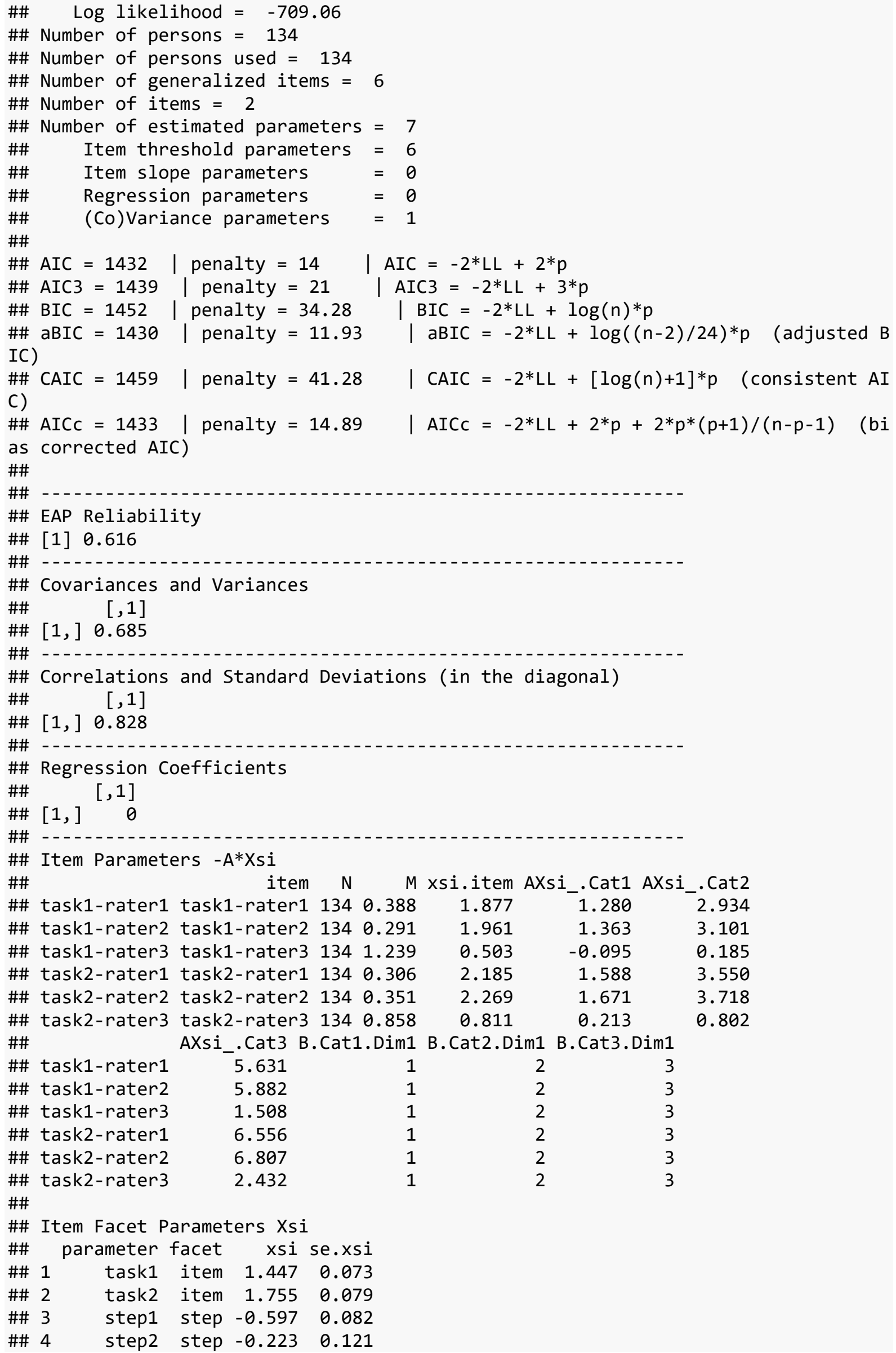




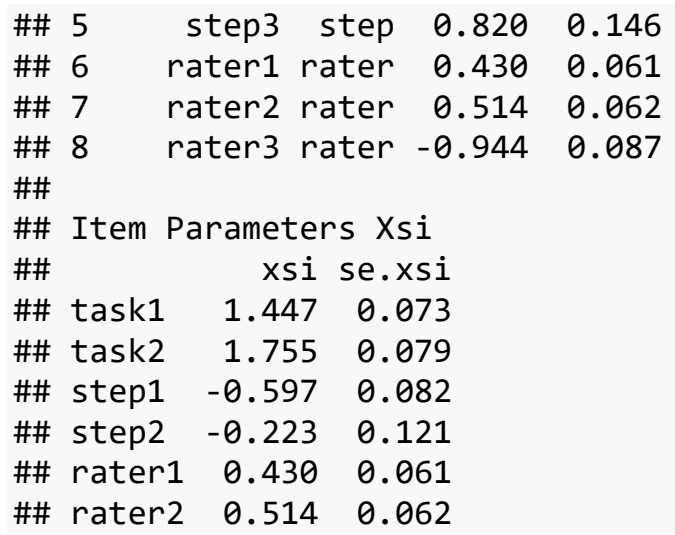

We can explore task, rating scale and rater parameters by examining the data frame called $x s i$. facets within fit list. There is also an interesting utility function called IRT. WrightMap. We can pass fit object to this function to get a construct map.

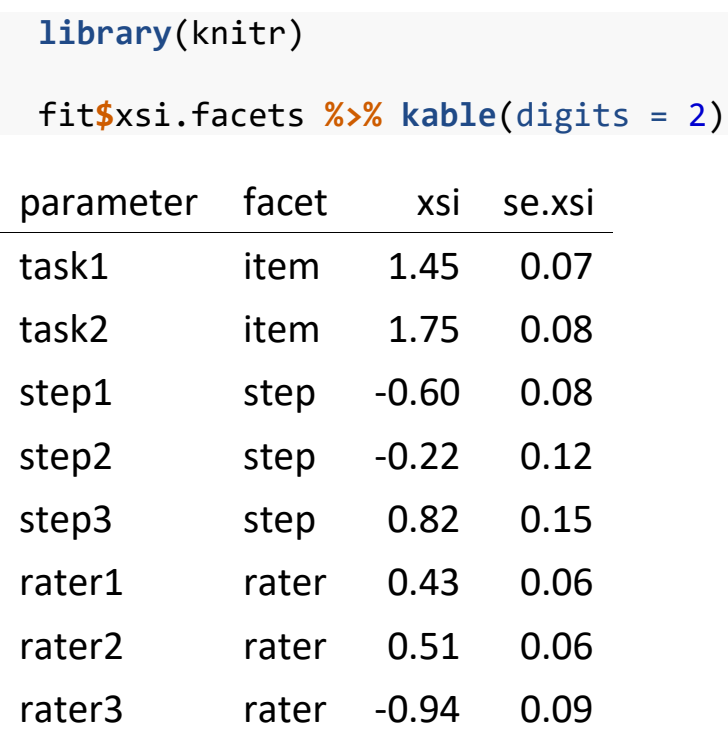




\section{Wright Map}

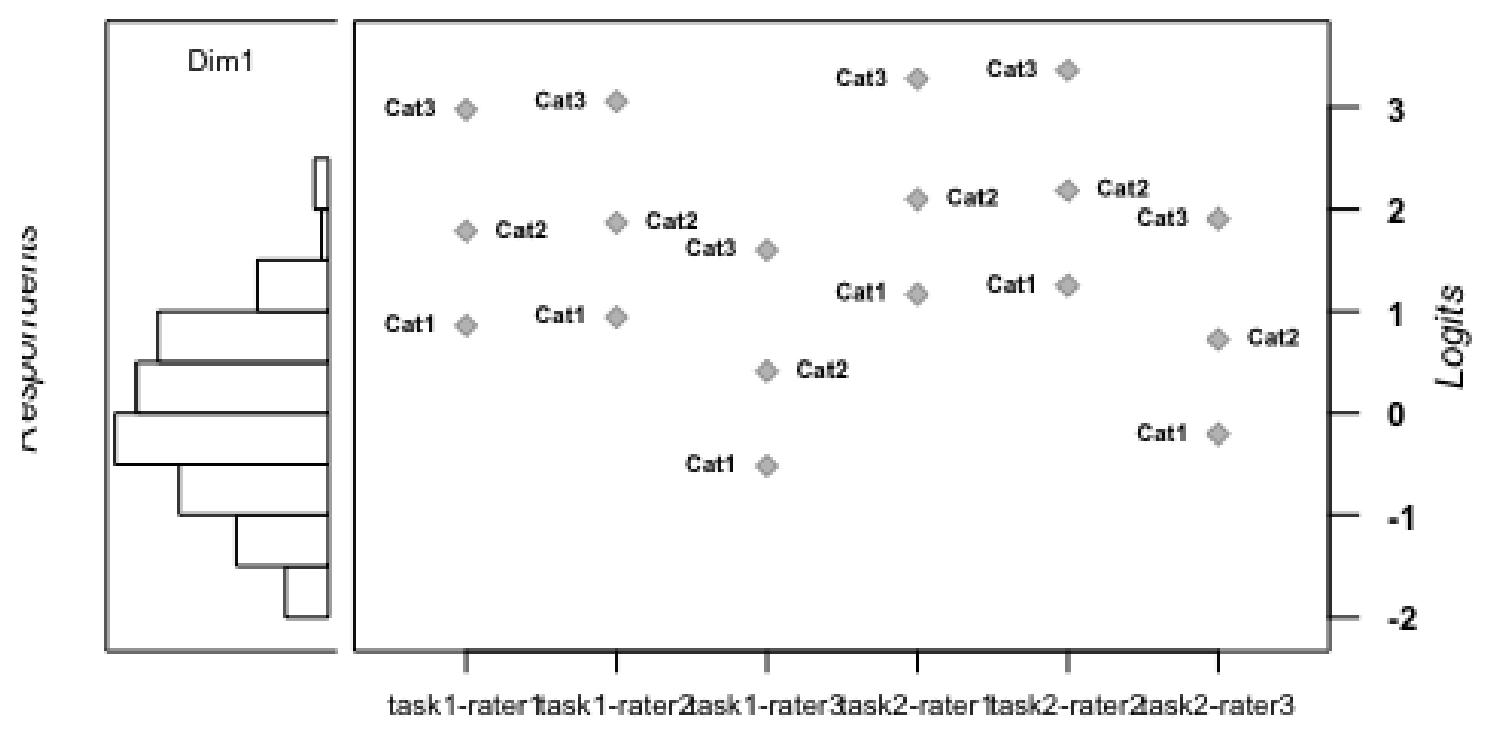

Items

Note that different software may use distinct estimations methods and parametrizations that will produce results in different metrics. Let's explore results from TAM vs FACETS. We exported estimated parameters from FACETS: subject's $\theta$, task's $\beta$ and rater's $\alpha$. Let's read these parameters from saved files via a web link.

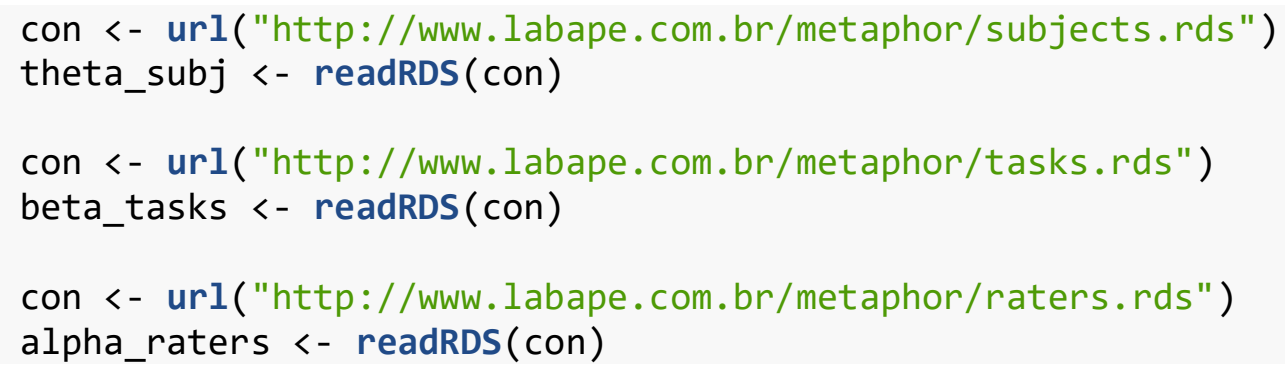

Now let's combine subject's theta from both programs in a data frame and visualize their relationship. We use pipe \% \% operator in order to run several commands in chain without the need to store its intermediate products. So the commands below do a lot of processing. We start from a data frame object theta_subj that contains data from FACETS; we then (1) select columns Subjects, Measure, S.E., T.Score, (2) transform the Subjects id's columns from character to numeric (3) join this data frame with the one produced by TAM using the by argument to inform which column contains the unique subject identifier, (4) finally we produce a scatterplot using ggplot graph.

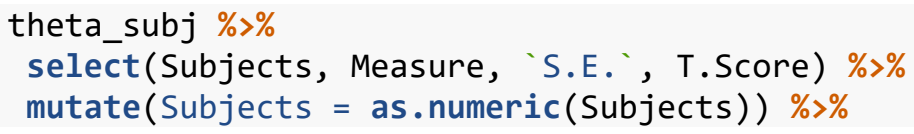


left_join( fit\$person, by=c("Subjects"="pid")) \%>\%

ggplot (aes ( $y=$ Measure, $x=E A P$, colour=`S.E.' $)$ ) +

geom_point () +

geom_smooth $($ method $=1 \mathrm{~m}$, se=FALSE) +

geom_abline (intercept $=0$, slope $=1$, color $="$ red", alpha=1/4) +

theme_light ()$+$

scale_x_continuous (breaks $=\operatorname{seq}(-3.5,2, .5)$, $\operatorname{limits}=c(-3.5,2))+$

scale_y_continuous $($ breaks $=\operatorname{seq}(-3.5,2, .5), \operatorname{limits}=c(-3.5,2))$

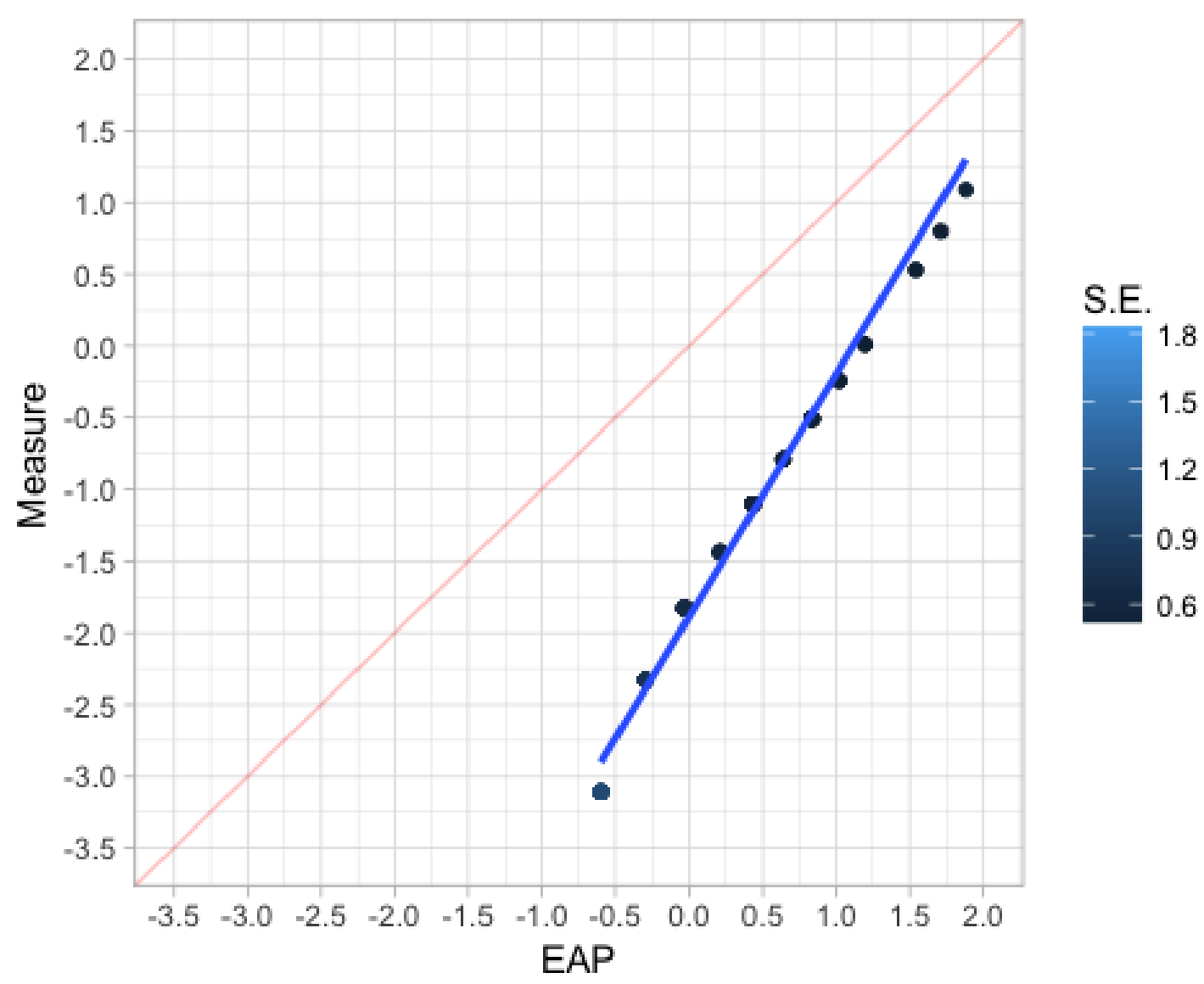

This figure shows FACETS subject's measure on the $y$-axis and TAM equivalent subject's EAP estimates on the x-axis. The correlation between these estimates are $r=.98$. We can see in this figure that they are nearly the same but come in a different metric. EAP estimates are centered on 0 . But in FACETS subject's $\theta^{\prime} s$ were not centered but all the other parameters were. Let's calculate the descriptive statistics of subjects $\theta^{\prime} s$. We repeat the code presented earlier up to Left_join. We then select variables and use a function from psych package called describe.

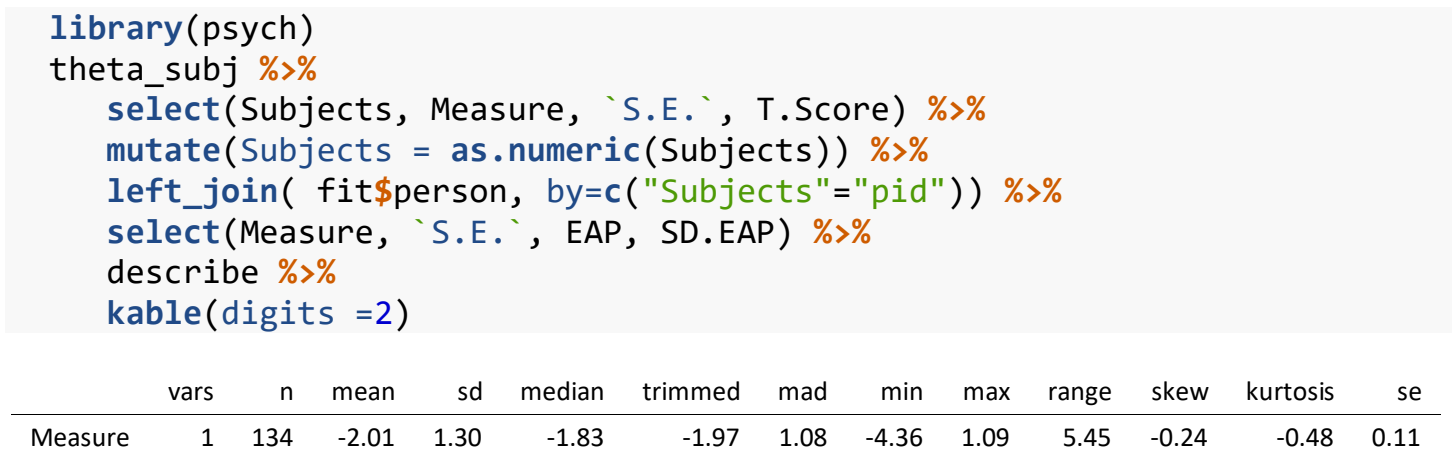




\begin{tabular}{|c|c|c|c|c|c|c|c|c|c|c|c|c|c|}
\hline S.E. & 2 & 134 & 0.84 & 0.42 & 0.66 & 0.76 & 0.16 & 0.51 & 1.84 & 1.33 & 1.60 & 1.19 & 0.04 \\
\hline EAP & 3 & 134 & 0.00 & 0.65 & -0.03 & -0.03 & 0.69 & -0.94 & 1.88 & 2.82 & 0.47 & -0.29 & 0.06 \\
\hline D.EAP & 4 & 134 & 0.51 & 0.06 & 0.50 & 0.51 & 0.06 & 0.41 & 0.61 & 0.20 & 0.18 & -1.04 & 0.01 \\
\hline
\end{tabular}

Therefore, we see that Measure from FACETS is 2 units below the metric of TAM presented as EAP. Also its standard deviation is 2 times higher. Let's consider the other parameters.

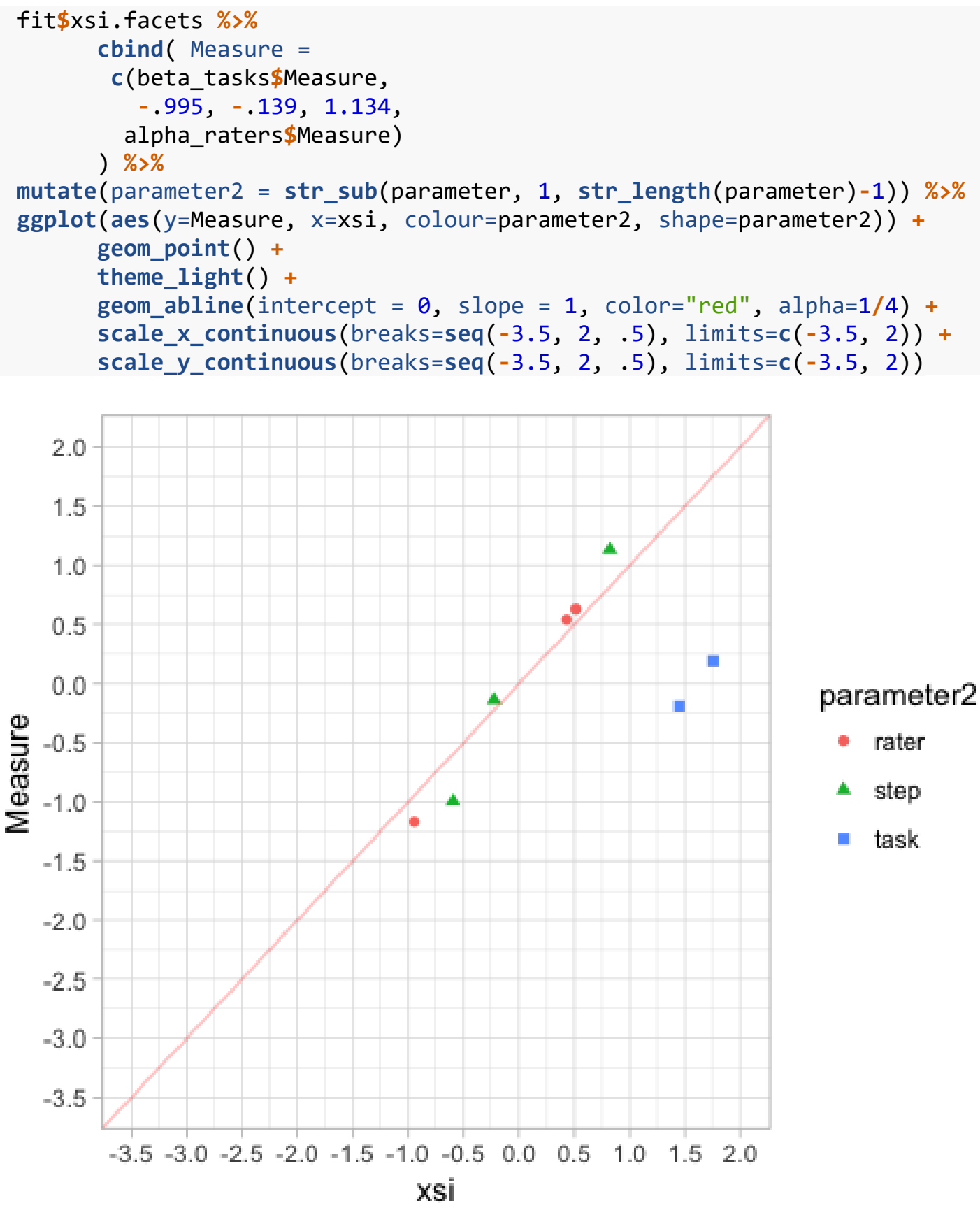

We can see in this figure that all parameters are linearly related. We can also see that task parameters are shifted. In FACETS we centered tasks, rater and category threshold parameters. We can see that they clustered around zero on the $y$-axis. We let theta vary freely and because items scores tend to be low, the average of theta is below zero $(\theta=$ -2). In TAM, rater, thresholds and theta parameters are centered, therefore tasks became more difficult. That's why we see tasks parameters shifted to the right in $\mathrm{x}$-axis. 
A very useful way to visualize these relationships is via construct maps. Construct maps shows figures representing (a) the distribution of subjects' $\theta$, (b) the distribution of items' $\beta$, (c) raters' $\alpha$, and (d) category probability curves that inform the rating scale structure and $\tau$ thresholds. Since all parameters are in the same metric it is possible to make comparisons inferring their relative contributions to the final score. In the next figure we see the construct map based on FACETS parameters. We use a function called

mfrm_construct_map. $R$ that takes as arguments vectors with thetas, raters alphas, tasks betas and tau treshodls.

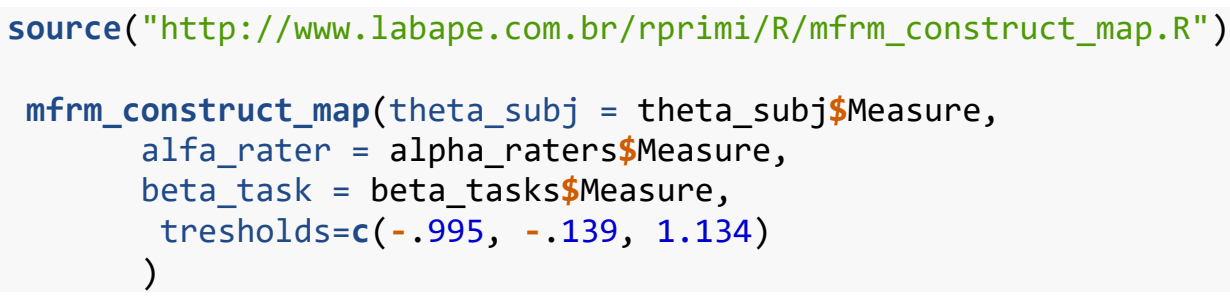

\section{Subject's $\theta$}

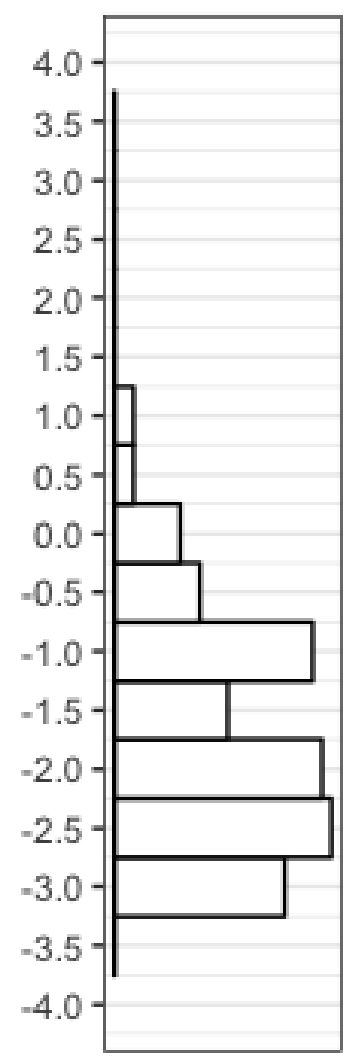

Item's $\beta$

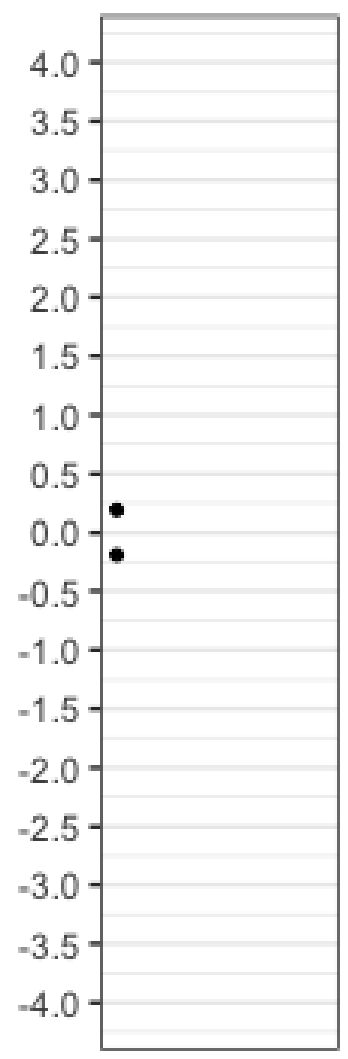

Rater's $\alpha$

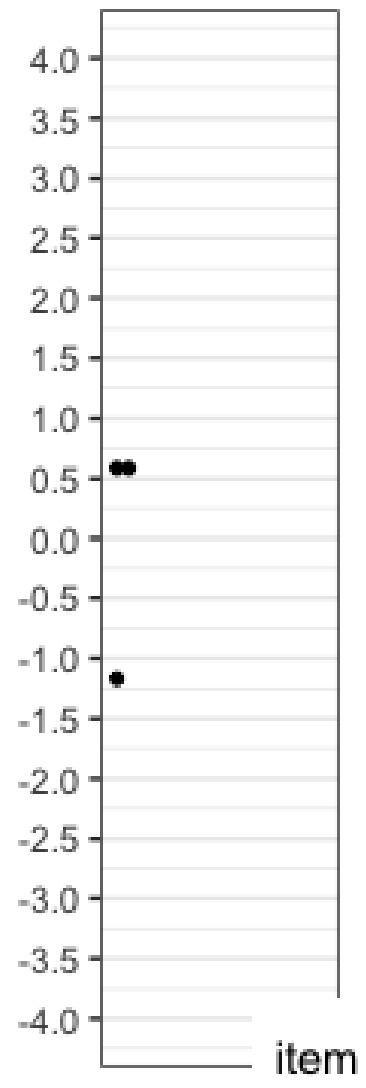

Scale's $\tau$

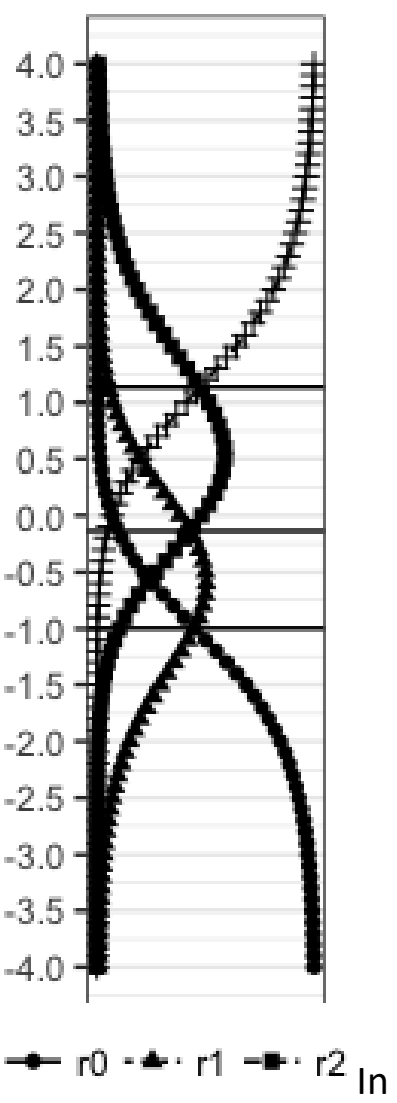

The next figure present parameters based on TAM.

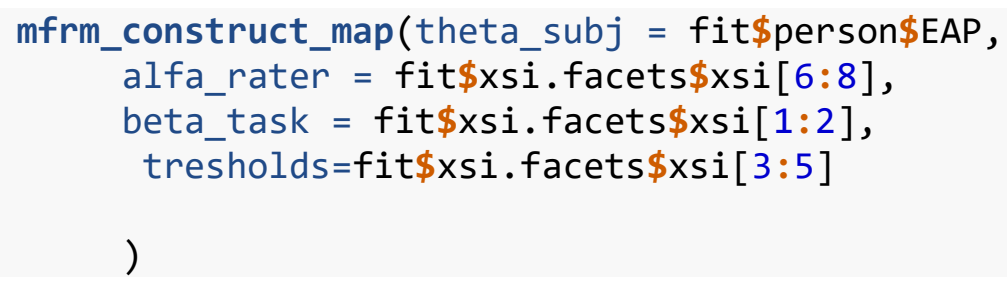


Subject's $\theta$

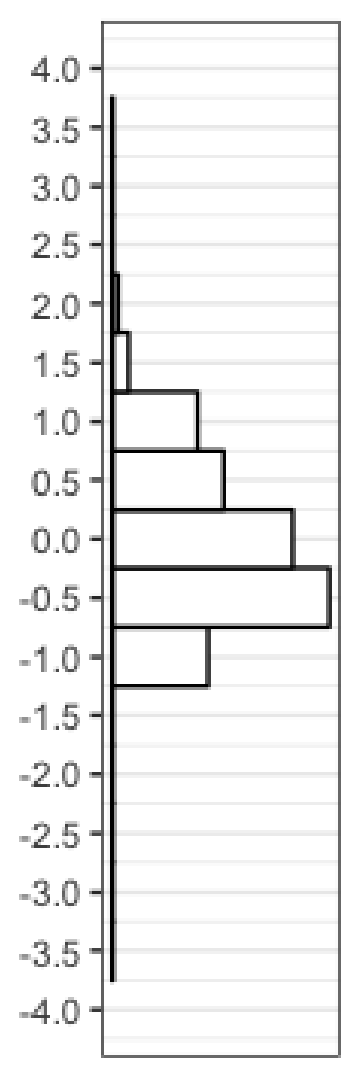

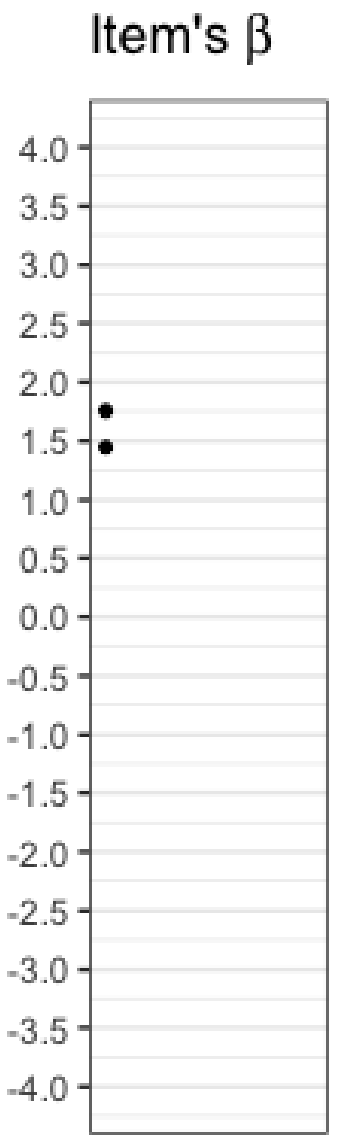

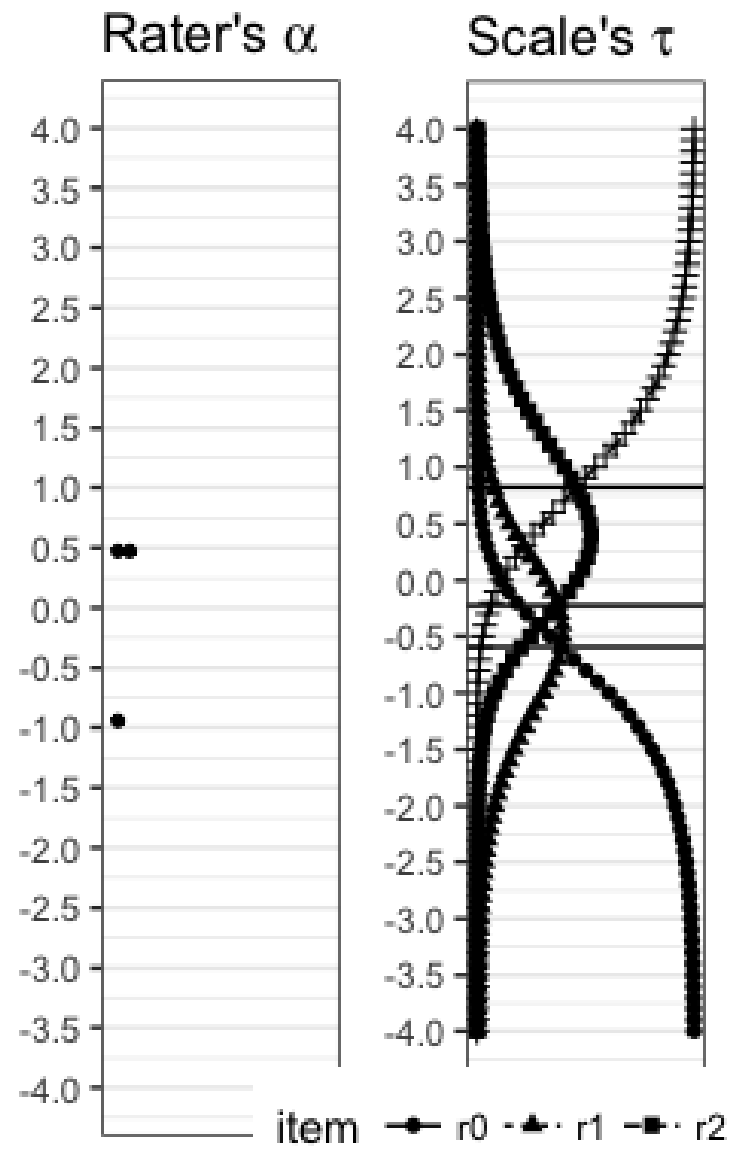

We can see how the different parametrizations operate. Although both programs present slightly different numbers, in both cases, the relative distance of persons and items are similar. The mean level of $\theta$ is always below items' $\beta$. This corresponds to the high prevalence of scores $0 / 1$ versus $2 / 3$. We can also see that the dispersion of parameters are lower in TAM as compared to FACETS. This is a consequence of the method TAM uses for estimation of theta, Expected a Posteriori (EAP), which shrinks the theta distribution towards zero. FACETS uses joint marginal maximum likelihood that doesn't shrink scores.

\section{Conclusion}

This tutorial presented a basic walk through to run Many Facet Rasch Models with creativity ratings on a divergent thinking task. FACETS is a commercial software with several useful outputs related to the most practical problems that we face when running such data analysis. It also has a lot of flexibility in testing different models like Differential Rater Functioning. TAM is an open source $\mathrm{R}$ package with a lot of flexibility for testing different models beyond the Rasch model families (see also Immer package Alexander Robitzsch \& Steinfeld, 2017). We showed a basic setup for creativity research that can produce equivalent results in both programs.

\section{References}

Robitzsch, A., \& Steinfeld, J. (2017). Immer: Item Response Models for Multiple Ratings. Retrieved from https://CRAN.R-project.org/package=immer 
Robitzsch, A., \& Steinfeld, J. (2018). Item response models for human ratings: Overview, estimation methods, and implementation in R. Psychological Test and Assessment Modeling, 60(1), 101-139.

Robitzsch, A., Kiefer, T., \& Wu, M. (2018). TAM: Test analysis modules. Retrieved from https://CRAN.R-project.org/package=TAM

Silvia, P. J., \& Beaty, R. E. (2012). Making creative metaphors: The importance of fluid intelligence for creative thought. Intelligence, 40(4), 343-351.

doi:10.1016/j.intell.2012.02.005

Wickham, H., \& Grolemund, G. (2017). R for Data Science: Import, Tidy, Transform, Visualize, and Model Data (1st ed.). O’Reilly Media, Inc. 